\title{
Constructing Minimum Deflection Fixture Arrangements Using Frame Invariant Norms
}

\author{
Qiao Lin, Joel W. Burdick, and Elon Rimon, Member, IEEE
}

\begin{abstract}
This paper describes a fixture planning method that minimizes object deflection under external loads. The method takes into account the natural compliance of the contacting bodies and applies to two-dimensional and three-dimensional quasirigid bodies. The fixturing method is based on a quality measure that characterizes the deflection of a fixtured object in response to unit magnitude wrenches. The object deflection measure is defined in terms of frame-invariant rigid body velocity and wrench norms and is therefore frame invariant. The object deflection measure is applied to the planning of optimal fixture arrangements of polygonal objects. We describe minimum-deflection fixturing algorithms for these objects, and make qualitative observations on the optimal arrangements generated by the algorithms. Concrete examples illustrate the minimum deflection fixturing method.
\end{abstract}

Note to Practitioners-During fixturing, a workpiece needs to not only be stable against external perturbations, but must also stay within a specified tolerance in response to machining or assembly forces. This paper describes a fixture planning approach that minimizes object deflection under applied work loads. The paper describes how to take local material deformation effects into account, using a generic quasirigid contact model. Practical algorithms that compute the optimal fixturing arrangements of polygonal workpieces are described and examples are then presented.

Index Terms-Fixture planning, frame-invariant norms, grasp quality measure, object deflection.

\section{INTRODUCTION}

$\mathbf{T}$ HIS PAPER is concerned with planning fixture arrangements of quasirigid workpieces. Fixturing plays an important role in manufacturing and assembly applications [6], [35], [47]. In these applications, a workpiece is fixtured with some preloading forces by fixturing elements, or fixels. The workpiece need not only be stable against external perturbations, but must also stay within a specified tolerance in response to machining or assembly forces [5], [18], [29]. It has been theoretically and experimentally shown that the deflection of fixtured objects due to contact compliance is a major source of geometric error in machining operations, and that fixel geometry and layout can significantly reduce such deflections [16], [17], [41]. This paper describes a fixture planning approach

Manuscript received June 19, 2004; revised June 1, 2005. This paper was recommended for publication by Associate Editor H. Ding and Editor M. Wang upon evaluation of the reviewers' comments.

Q. Lin is with the Department of Mechanical Engineering, Columbia University, New York, NY 10027 USA (e-mail: q12134@ columbia.edu).

J. W. Burdick is with the Department of Mechanical Engineering, California Institute of Technology, Pasadena, CA 91125 USA (e-mail: jwb@robotics.caltech.edu).

E. Rimon is with the Department of Mechanical Engineering, The Technion, Israel Institute of Technology, Haifa 32000, Israel (e-mail: elon@ robby.technion.ac.il).

Digital Object Identifier 10.1109/TASE.2006.872104 that minimizes object deflection under applied work loads. Loosely speaking, the deflection of a fixtured object is the average or maximal displacement of the object's individual particles. Using frame-invariant rigid body velocity and wrench norms, we characterize the deflection of a fixtured object under a general class of quasirigid contact models. Based on this characterization, we define a frame-invariant object deflection measure and apply the measure to optimal fixture planning of polygonal objects.

The grasping and fixturing literature has a long tradition of using quality measures to quantify and select optimal contact arrangements. For example, the authors in [26] define as a quality measure the smallest singular value of the matrix whose columns consist of the generating wrenches (i.e., wrenches due to unit finger forces). Subsequent quality measures compute the maximal wrench ball inscribed in the convex hull of the generating wrenches [9], [25], [42]. Other quality measures either minimize the worst-case finger forces needed to balance a collection of pure forces acting on the object [31], or treat forces and torques lexicographically [34]. Another notable approach is based on assessing the static stability margin afforded by a given contact arrangement. This approach was pioneered by the authors in [24], and subsequently extended in [1], [4], [13], [21], [45], [49]. An alternative quality measure, suggested by Nakamura et al. [36], considers the largest allowable dynamic perturbation to the contact positions that does not cause slippage. However, all of these quality measures assume perfectly rigid bodies which do not experience any contact deformation in response to applied loads. Moreover, some of these quality measures employ ill-defined wrench norms. This paper clarifies the meaning of frame-invariant velocity and wrench norms, and applies these notions to define a frame-invariant object deflection measure.

In contrast with grasping applications, industrial fixtures must withstand work loads as high as $5000 \mathrm{lbf}$ [14], and the precision of such fixtures is highly affected by contact compliance [47]. The literature contains relatively few compliant quality measures, all of which rely on the grasp stiffness matrix [2]. For example, the authors [7] consider the eigenvalues of the grasp stiffness matrix, while Lin et al. consider the behavior of the stiffness matrix on certain invariant subspaces [29]. Another example is the work of Donoghue et al. [8], who propose, as a quality measure, a weighted sum of displacement components which are determined by the grasp stiffness matrix. However, the existing quality measures give only an indirect indication on the amount of object deflection under workloads. The quality measure described in this paper directly characterizes the deflection of object particles under workloads. Prior efforts at as- 
sessing object deflection (as well as fixture stresses) are based on finite-element analysis [33], [39], [44]. While this approach offers excellent accuracy, it has drawbacks. The contact compliance models relating fixture reaction forces to object displacement can only be obtained numerically. Thus, while such numerical methods are useful for verifying existing fixture designs, they are not well suited for early planning stages when workpiece designs and process plans are frequently modified. This paper provides a direct characterization of object deflection in a lumped parameter form which is suitable for automated fixture planning.

The paper is organized as follows. The next section reviews the modeling of compliant contact arrangements using the fixture stiffness matrix. Section III discusses the notions of frameinvariant velocity and wrench norms. These notions are part of a general theory on objective metric functions [28], which is applied here to the formulation of a frame-invariant object deflection measure. In Section IV, we characterize the deflection of a fixtured object in response to unit magnitude wrenches. Based on this measure, we describe in Section V practical algorithms that compute the globally optimal three-contact and four-contact fixturing arrangements of polygonal workpieces. These global optimization algorithms are significant, since the underlying optimization problem is nonconvex and nonlinear. Examples are then presented, and qualitative properties of the optimal fixtures are described. In the concluding section, we discuss future research issues, which include the development of a high-precision fixturing system [3].

\section{Modeling OF COMPLiANT FiXTURES}

This section briefly reviews the stiffness-matrix model for compliant fixtures. A fixture arrangement consists of an object $\mathcal{B}$, which is, in general, three-dimensional (3-D), contacted by $k$ stationary fixels $\mathcal{A}_{1}, \ldots, \mathcal{A}_{k} \cdot{ }^{1}$ We assume that the bodies are quasirigid (i.e., deformations due to material compliance are localized at the contacts). Elasticity theory [23], [43] suggests that this is an excellent assumption as long as the bodies are not made of exceptionally soft material and do not contain slender substructures.

The overall motion of $\mathcal{B}$ relative to the stationary fixels can be efficiently described in $\mathcal{B}$ 's configuration space as follows. Let $\mathcal{F}_{W}$ be a stationary world frame, and let $\mathcal{F}_{B}$ be a body frame fixed to $\mathcal{B}$. A configuration of $\mathcal{B}$ is a pair $(d, R)$, where $d \in \mathbb{R}^{3}$ and $R \in S O(3)$ are the position and orientation of $\mathcal{F}_{B}$ relative to $\mathcal{F}_{W}$. The set of all configurations, denoted as $\mathcal{C}$, is $\mathcal{B}$ 's configuration space (or c-space). We parametrize $\mathcal{C}$ using hybrid coordinates $q=(d, \theta) \in \mathbb{R}^{3} \times \mathbb{R}^{3}$, where $\theta \in \mathbb{R}^{3}$ parametrizes $S O(3)$ by exponential coordinates. The tangent space to $\mathcal{C}$ at $q$, denoted as $T_{q} \mathcal{C}$, is the set of all tangent vectors, or velocities of $\mathcal{B}$, at $q$. Tangent vectors represent instantaneous displacements of $\mathcal{B}$ and can approximate small displacements of the object. In hybrid coordinates, tangent vectors take the form $\dot{q}=(v, \omega)$,

\footnotetext{
${ }^{1}$ This paper focuses on completely loaded fixtures, in which all active fixels are immobilized upon completion of the fixture loading process. Hence, there is no loss of generality in assuming stationary fixels.
}

where $v$ and $\omega$ are linear and angular velocities of $\mathcal{F}_{B}$ relative to $\mathcal{F}_{W}$. (Tangent vectors represent velocities $\dot{q}$, or small displacements $\dot{q} d t$ where $d t$ is a small time interval. We use the latter interpretation in this paper while notationaly dropping $d t$.) The wrench space at $q$, denoted $T_{q}^{*} \mathcal{C}$, is the set of all wrenches (or covector) acting on $\mathcal{B}$ at $q$. A wrench takes the form $\boldsymbol{w}=(f, \tau)$ in hybrid coordinates, where $f$ is the force acting on $\mathcal{B}$ and $\tau$ is the torque. When $\mathcal{B}$ is a 2 -D object, c-space is parameterized by $q=(d, \theta) \in \mathbb{R}^{2} \times \mathbb{R}$. In this case, tangent vectors and wrenches are written as $\dot{q}=(v, \omega) \in \mathbb{R}^{2} \times \mathbb{R}$ and $\boldsymbol{w}=(f, \tau) \in \mathbb{R}^{2} \times \mathbb{R}$.

Consider a compliant fixture in which an object $\mathcal{B}$ is held at an equilibrium configuration $q_{0} \in \mathcal{C}$. That is, in the absence of external wrenches, forces applied by the fixels produce zero net wrench on $\mathcal{B}$. When subjected to an external wrench $\boldsymbol{w} \in T_{q}^{*} \mathcal{C}$, the object experiences a small displacement. To first order, this displacement can be approximated by a velocity $\dot{q} \in T_{q} \mathcal{C}$, which is related to $\boldsymbol{w}$ by

$$
\boldsymbol{w}=K \dot{q} \quad \text { or } \quad \dot{q}=C \boldsymbol{w}
$$

where $K$ is the fixture stiffness matrix, and $C=K^{-1}$ is the fixture compliance matrix. If $K$ and $C$ are positive definite, the fixture is stable.

The object's c-space can be described using alternative world and object frames $\overline{\mathcal{F}}_{W}$ and $\overline{\mathcal{F}}_{B}$ as follows. Let $\overline{\mathcal{F}}_{W}$ be displaced from $\mathcal{F}_{W}$, and $\overline{\mathcal{F}}_{B}$ from $\mathcal{F}_{B}$ by constant transformations $\left(d_{w}, R_{w}\right)$ and $\left(d_{b}, R_{b}\right)$. Then, the equilibrium configuration will have some new coordinates $\bar{q}_{0}$. The applied wrench and resulting object displacement will also have new coordinates, which are related to the original coordinates by $\bar{w}=T^{T} \boldsymbol{w}$ and $\dot{q}=T \dot{\bar{q}}$, respectively. Thus, the stiffness matrix obeys the transformation rule

$$
\bar{K}=T^{T} K T
$$

where $\bar{K}$ is the stiffness matrix associated with the frames $\overline{\mathcal{F}}_{W}$ and $\overline{\mathcal{F}}_{B}$. Here, in hybrid coordinates, the transformation matrix is given for the 2-D and 3-D cases by

$$
T_{6 \times 6}=\left(\begin{array}{cc}
R_{w} & R_{w} \widehat{\bar{R} d_{b}} \\
0 & R_{w}
\end{array}\right) \quad \text { and } \quad T_{3 \times 3}=\left(\begin{array}{cc}
R_{w} & J \bar{R} d_{b} \\
0 & 1
\end{array}\right)
$$

where $\widehat{v}$ is the $3 \times 3$ skew-symmetric matrix such that $\widehat{v}=v \times x$ for $x \in \mathbb{R}^{3}, \bar{R}$ is the orientation of $\overline{\mathcal{F}}_{B}$ relative to $\overline{\mathcal{F}}_{W}$ at $\bar{q}$, and $J=\left(\begin{array}{cc}0 & 1 \\ -1 & 0\end{array}\right)$.

While the computation of the fixture stiffness matrix $K$ is beyond the scope of this paper, $K$ can be computed from any lumped-parameter contact compliance model which is based on the quasirigid assumption. Examples include [8], [12], [20], [30], [37], [38], [48], and [50]. These examples are based on linear spring models [19] or the classical Hertz model [22]. However, it is important to note that (1) is generally applicable to quasirigid fixtures and is not restricted to these specific approaches. 


\section{FRAME-INVARIANT VELOCITY AND WRENCH NORMS}

The object deflection measure is based on frame-invariant velocity and wrench norms ${ }^{2}$ which are introduced in this section.

\section{A. Frame-Invariant Velocity Norms}

Consider a $3-D$ object $\mathcal{B}$ with a configuration space $\mathcal{C}$. Once the tangent space $T_{q} \mathcal{C}$ is parametrized by $\dot{q} \in \mathbb{R}^{6}$, one can use the Euclidean norm of $\mathbb{R}^{6}$ as a velocity norm. However, this norm does not satisfy the important property of frame invariance - the same physical velocity of $\mathcal{B}$ will have different norms depending on the body and world frames being used to parametrize c-space. Moreover, the Euclidean norm combines translational and rotational velocities in an ad-hoc way. We now describe two rigid-body velocity norms which are free of both problems. The two norms belong to a general family of norms whose definition requires some notation. Let $\mathcal{B}(q)$ denote the region of $\mathbb{R}^{3}$ occupied by the object at a configuration $q$. Let $x$ denote points of $\mathcal{B}(q)$ described in $\mathcal{F}_{W}$, and let $r$ denote points of $\mathcal{B}$ described in its body frame $\mathcal{F}_{B}$. The two representations are related by the rigid-body transformation $x=R(\theta) r+d$, where $q=(d, \theta)$. When $\mathcal{B}$ moves along a c-space trajectory $q(t)$, a fixed body point $r$ traces a curve $x(t)$ whose velocity is given by $\dot{x}=\omega \times R r+v$, where $\dot{q}=(v, \omega)$.

Definition 1: Let $\nu: \mathcal{B} \rightarrow \mathbb{R}$ be a non-negative weighting function such that $\int_{\mathcal{B}} \nu(r) d r=1$. Let $\dot{q}=(v, \omega)$ be a velocity of $\mathcal{B}$ at $q$, and let $p>1$ be an integer. The $\boldsymbol{p}$ velocity norm of $\mathcal{B}$ with respect to $\nu$ is

$$
\begin{aligned}
\|\dot{q}\|_{p} & =\left(\int_{\mathcal{B}(q)} \nu(x)\|\dot{x}\|^{p} d x\right)^{\frac{1}{p}} \\
& =\left(\int_{\mathcal{B}} \nu(r)\|\omega \times R r+v\|^{p} d r\right)^{\frac{1}{p}}
\end{aligned}
$$

where $\|\cdot\|$ is the usual Euclidean norm on $\mathbb{R}^{3}$, and $R$ is the orientation matrix of $\mathcal{B}$ at $q$.

We verify the norm properties of two special $p$-norms which are most relevant for assessing object deflection. The first is the root-mean-square or rms velocity norm.

Definition 2: The rms velocity norm of $\mathcal{B}$ with respect to $\nu$ is the 2-norm given by

$$
\begin{aligned}
\|\dot{q}\|_{\mathrm{rms}} & =\left(\int_{\mathcal{B}(q)} \nu(x)\|\dot{x}\|^{2} d x\right)^{\frac{1}{2}} \\
& =\left(\int_{\mathcal{B}} \nu(r)\|\omega \times R r+v\|^{2} d r\right)^{\frac{1}{2}} .
\end{aligned}
$$

The rms norm can be written in the following equivalent form. Using the identities $R\left(v_{1} \times v_{2}\right)=R v_{1} \times R v_{2}$ and $R^{T} R=I$,

${ }^{2}$ Recall that a norm on a vector space $V$ is a function $\|\cdot\|: V \rightarrow \mathbb{R}$ such that $\|x\| \geqslant 0$ and $\|x\|=0$ only at $x=0$ (positive definiteness), $\|\alpha x\|=|\alpha|\|x\|$ (linear homogeneity), and $\|x+y\| \leqslant\|x\|+\|y\|$ (triangle inequality) for all $x, y \in V$ and $\alpha \in \mathbb{R}$. one can write $\|\omega \times R r+v\|=\left\|\left(R^{T} \omega\right) \times r+R^{T} v\right\|$. Hence, the rms norm is given by

$$
\begin{aligned}
\|\dot{q}\|_{\text {rms }} & =\left(\dot{q}^{T} \mathcal{R} M \mathcal{R}^{T} \dot{q}\right)^{\frac{1}{2}} \text { where } \\
\mathcal{R} & =\operatorname{diag}(R, R) \text { and } \\
M & =\int_{\mathcal{B}} \nu(r)\left(\begin{array}{cc}
I & \widehat{r}^{T} \\
\widehat{r} & -\widehat{r}^{2}
\end{array}\right) d r .
\end{aligned}
$$

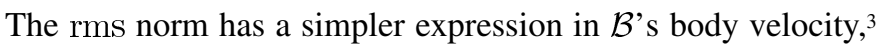

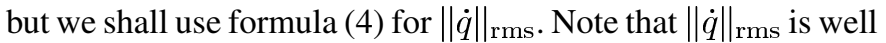
defined, since the matrix $M$, called the weighting matrix, is positive semidefinite. The verification that $\|\dot{q}\|_{\text {rms }}$ is a norm is now straightforward. The matrix $M$ is generically positive definite and, in this case, (4) is positive definite, linearly homogeneous in $\dot{q}$, and satisfies the triangle inequality. The intuition behind the rms norm is as follows. When $\nu$ is mass density, the rms norm is simply the kinetic energy of $\mathcal{B}$. However, a more interesting interpretation can be given from a purely kinematic point of view. The function $\nu$ can be interpreted as a weighting function for $\mathcal{B}$ 's pointwise velocities (assigned according to process precision constraints), and $\|\dot{q}\|_{\mathrm{rms}}$ is a weighted average of $\mathcal{B}$ 's pointwise velocities. This interpretation is used below to quantify the average deflection of $\mathcal{B}$ 's points in response to an applied load. The following lemma asserts the frame invariance of the rms norm (Appendix A).

Lemma 1: The rms velocity norm is invariant with respect to the choice of world and body frames.

Similar to kinetic energy, the rms norm has a simple expression when the origin of $\mathcal{F}_{B}$ is chosen at $\mathcal{B}$ 's centroid with respect to the weighting function $\nu$. (The centroid is the point $\int_{\mathcal{B}} r \nu(r) d r$.) For a 2-D object, the rms norm becomes $\|\dot{q}\|_{\text {rms }}=$ $\left(v^{T} v+\rho^{2} \omega^{2}\right)^{1 / 2}$, where $\rho=\left(\int_{\mathcal{B}} \nu(r)\|r\|^{2} d r\right)^{1 / 2}$. The scalar $\rho$ is $\mathcal{B}$ 's radius of gyration when $\nu$ is mass density. The second norm measures the maximal rather than average velocity of $\mathcal{B}$ 's points.

Definition 3: The max velocity norm of $\mathcal{B}$ with respect to the weighting function $\nu$ is the $\infty$-norm given by

$$
\begin{aligned}
\|\dot{q}\|_{\text {max }} & =\max _{x \in \mathcal{B}(q)} \\
\{\nu(x)\|\dot{x}\|\} & =\max _{r \in \mathcal{B}}\{\nu(r)\|\omega \times R r+v\|\} .
\end{aligned}
$$

The max norm satisfies the inequality $\|\dot{q}\|_{\max } \geqslant\|\dot{q}\|_{\mathrm{rms}}$ for all $\dot{q}$ and weighting functions $\nu$. Since $\|\omega \times R r+v\|=$ $\left\|\left(R^{T} \omega\right) \times r+R^{T} v\right\|$, we can write the max norm as

$$
\begin{aligned}
\|\dot{q}\|_{\max } & =\max _{r \in \mathcal{B}}\left\{\left(\dot{q}^{T} \mathcal{R} N(r) \mathcal{R}^{T} \dot{q}\right)^{\frac{1}{2}}\right\} \text { where } \\
\mathcal{R} & =\operatorname{diag}(R, R) \text { and } \\
N(r) & =\nu^{2}(r)\left(\begin{array}{cc}
I & \widehat{r}^{T} \\
\widehat{r} & -\widehat{r}^{2}
\end{array}\right) .
\end{aligned}
$$

Note that $\|\dot{q}\|_{\max }$ is well defined, since the matrix $N$ is positive semi-definite. The following lemma specifies the precise condition under which $\|\dot{q}\|_{\max }$ is a norm (Appendix A).

Lemma 2: If $\mathcal{B}$ is a 2-D object, $\|\dot{q}\|_{\max }$ is a norm when $\nu$ is strictly positive at two points of $\mathcal{B}$. If $\mathcal{B}$ is a $3-\mathrm{D}$ object, $\|\dot{q}\|_{\max }$

${ }^{3}$ Let $\dot{q}_{b}=\left(v_{b}, \omega_{b}\right)$ be $\mathcal{B}$ 's body velocity. Then, $\left(v_{b}, \omega_{b}\right)=\left(R^{T} v, R^{T} \omega\right)$, hence $\left\|\dot{q}_{b}\right\|_{\mathrm{rms}}=\left(\int_{\mathcal{B}} \nu(r)\left\|\omega_{b} \times r+v_{b}\right\|^{2} d r\right)^{1 / 2}=\left(\dot{q}_{b}^{T} M \dot{q}_{b}\right)^{1 / 2}$. 
is a norm when $\nu$ is strictly positive at three noncollinear points of $\mathcal{B}$.

The arguments used to prove frame invariance of the rms norm (Lemma 1) also imply frame invariance of the max norm. The computation of the max norm becomes simple if one uses screw coordinates for $\dot{q}$ [32]. Suppose for simplicity that $\nu$ is a uniform weighting function of unity. If $\dot{q}$ is pure translation, then $\dot{q}=(v, 0)$ and $\|\dot{q}\|_{\max }=\|v\|$. Otherwise, $\dot{q}$ can be written as $\dot{q}=\alpha(v, \omega)$, where $\alpha>0$ and $\|\omega\|=1$. Using this notation, the velocity of a 3-D body can be regarded as an instantaneous screw motion with magnitude $\alpha$, pitch $h=v \cdot \omega$, and screw axis $\ell=\left\{R^{T}(v \times \omega+\lambda \omega): \lambda \in \mathbb{R}\right\}$. That is, $\dot{q}$ is an instantaneous rotation of magnitude $\alpha$ about $\ell$, accompanied by an instantaneous translation along $\ell$ by an amount $\alpha h$. Using screw coordinates, the max norm is given by $\|\dot{q}\|_{\max }=\alpha\left(\rho_{\max }^{2}+(v \cdot \omega)^{2}\right)^{1 / 2}$, where $\rho_{\max }$ is the maximal distance from the screw axis $\ell$ to $\mathcal{B}$ 's points. In the 2-D case, $\dot{q}$ simplifies to a rotation about $\mathcal{B}$ 's instantaneous center of rotation, a point given by $p=J R^{T} v$, where $J=\left(\begin{array}{cc}0 & 1 \\ -1 & 0\end{array}\right)$. In this case, $\|\dot{q}\|_{\max }=\alpha \rho_{\max }$, where $\rho_{\text {max }}$ is now the maximal distance from $p$ to $\mathcal{B}$ 's points.

Finally, one can choose a finite number of object points which are critical for precision monitoring, and only use these points in the definition of the rms and max norms. More precisely, let $r_{1}, \ldots, r_{m}$ be points on $\mathcal{B}$. Then, one can define a weighting function $\nu(r)=\sum_{i=1}^{m} \nu_{i} \delta\left(r-r_{i}\right)$, where $\delta$ denotes the Dirac delta function and $\sum_{i=1}^{m} \nu_{i}=1$. Under this choice, the two norms become $\|\dot{q}\|_{\text {rms }}=\left(\sum_{i=1}^{m} \nu_{i}\left\|\dot{x}_{i}\right\|^{2}\right)^{1 / 2}$ and $\|\dot{q}\|_{\max }=$ $\max _{1 \leqslant \mathrm{i} \leqslant \mathrm{m}}\left\{\nu_{\mathrm{i}}\left\|\dot{\mathrm{x}}_{\mathrm{i}}\right\|\right\}$, where $\dot{x}_{i}=\left[I_{3}-\widehat{r}_{i}\right] \dot{q}$ in the 3-D case, and $\dot{x}_{i}=\left[I_{2}-J r_{i}\right] \dot{q}$ in the 2-D case. We use the discretized norms below to compute minimum-deflection fixtures of polygonal objects.

\section{B. Frame-Invariant Wrench Norms}

A wrench acting on $\mathcal{B}$ at a configuration $q$ is formally a covector $\boldsymbol{w} \in T_{q}^{*} \mathcal{C}$ which acts linearly on tangent vectors $\boldsymbol{w}$ : $T_{q} \mathcal{C} \rightarrow \mathbb{R}$. A covector norm formalizes the notion of wrench magnitude. Similar to the Euclidean tangent vector norm, a Euclidean covector norm on a parametrization of $T_{q}^{*} \mathcal{C}$ by $\mathbb{R}^{6}$ is not frame invariant. A better approach is to start with a frame-invariant velocity norm and use this norm to induce a frame-invariant wrench norm. A standard procedure for generating covector norms from tangent vector norms is as follows. Given a velocity norm $\|\cdot\|_{p}$ and a covector $\boldsymbol{w}$, the dual wrench norm of $\boldsymbol{w}$, denoted $\||\boldsymbol{w}|\|_{p}$ is given by

$$
\|\boldsymbol{w} \mid\|_{p}=\max _{\|\dot{\mathrm{q}}\|_{\mathrm{p}}=1}\{\boldsymbol{w} \cdot \dot{\mathrm{q}} \cdot\}
$$

Note that the frame invariance of the dual wrench norm automatically follows from the frame invariance of the velocity norm. The following lemma applies this procedure to the rms velocity norm. ${ }^{4}$

\footnotetext{
${ }^{4}$ The procedure can also be applied to the max velocity norm, but the rms wrench norm is much simpler to compute [27].
}

Lemma 3: Let the matrix $M$ in $\|\dot{q}\|_{\mathrm{rms}}=\left(\dot{q}^{T} M \dot{q}\right)^{1 / 2}$ be positive definite. Then, the dual wrench norm induced by $\|\dot{q}\|_{\text {rms }}$, denoted $\left|\|\boldsymbol{w} \mid\|_{\mathrm{rms}}\right.$, is given by

$$
\||\boldsymbol{w}|\|_{\mathrm{rms}}=\left(\boldsymbol{w}^{T} M^{-1} \boldsymbol{w}\right)^{\frac{1}{2}} \text { where } M=\int_{\mathcal{B}} \nu(r)\left(\begin{array}{cc}
I & \widehat{r}^{T} \\
\widehat{r} & -\widehat{r}^{2}
\end{array}\right) d r .
$$

Moreover, $\||\boldsymbol{w}|\|_{\mathrm{rms}}$ is invariant with respect to the choice of world and body frames.

Proof: Let $\|x\|$ denote the usual Euclidean norm, and let $P$ be a symmetric matrix. In general, the maximum value of a quadratic form $x^{T} P x$ over $\|x\|=1$ is $\lambda_{\max }(P)$, the maximal eigenvalue of $P$. In our case, \|\| $\boldsymbol{w}\|\|_{\text {rms }}^{2}=\max \left\{(\boldsymbol{w} \cdot \dot{\mathrm{q}})^{2}\right\}$ over $\|\dot{q}\|_{\mathrm{rms}}=\left(\dot{q}^{T} M \dot{q}\right)^{1 / 2}=1$. Since $M$ is positive definite, we can define $x=M^{1 / 2} \dot{q}$ and write the rms norm as $\|\boldsymbol{w} \mid\|_{\mathrm{rms}}^{2}=\max \left\{\mathrm{x}^{\mathrm{T}} \mathrm{M}^{-1 / 2} \boldsymbol{w} \boldsymbol{w}^{\mathrm{T}} \mathrm{M}^{-1 / 2} \mathrm{x}\right\}$ over $\|x\|=1$. The matrix $M^{-1 / 2} \boldsymbol{w} \boldsymbol{w}^{T} M^{-1 / 2}$ is positive semidefinite and has a rank of unity. Hence, it has a single nonzero eigenvalue which is also its maximal eigenvalue. This eigenvalue is given by

$$
\left(M^{-\frac{1}{2}} \boldsymbol{w} \boldsymbol{w}^{T} M^{-\frac{1}{2}}\right) M^{-\frac{1}{2}} \boldsymbol{w}=\left(\boldsymbol{w}^{T} M^{-1} \boldsymbol{w}\right) M^{-\frac{1}{2}} \boldsymbol{w} .
$$

Hence, $\|\boldsymbol{w} \mid\|_{\mathrm{rms}}^{2}=\lambda_{\max }\left(M^{-1 / 2} \boldsymbol{w} \boldsymbol{w}^{T} M^{-1 / 2}\right)=$ $\boldsymbol{w}^{T} M^{-1} \boldsymbol{w}$.

The rms wrench norm is used below to define frame-invariant unit wrench balls. We conclude this section with a remark on the physical interpretation of the rms wrench norm [27]. Imagine that $\boldsymbol{w}=(f, \tau)$ is generated by a system of distributed forces, denoted $\mathrm{f}(r)$, where $r \in \mathcal{B}$ with respect to the weighting function $\nu$. That is, $f=\int_{\mathcal{B}} \nu(r) \mathrm{f}(r) d r$ and $\tau=\int_{\mathcal{B}} \nu(r) r \times \mathrm{f}(r) d r$. Let $\mathcal{D}(\boldsymbol{w})$ denote the collection of all force distributions such that $\int_{\mathcal{B}} \nu(r)\|\mathrm{f}(r)\|^{2} d r$ is finite. Then

$$
\||\boldsymbol{w}|\|_{\mathrm{rms}}=\inf _{\mathrm{f} \in \mathcal{D}(\boldsymbol{w})}\left\{\left(\int_{\mathcal{B}} \nu(r)\|\mathrm{f}(r)\|^{2} d r\right)^{\frac{1}{2}}\right\} .
$$

In words, the rms norm of a wrench $\boldsymbol{w}$ is the infimum rms average over all force distributions that generate the net wrench $\boldsymbol{w}$.

\section{ObJect Deflection Quality Measure}

Before introducing the deflection quality measure, recall that the displacement of a fixtured object in response to a wrench $\boldsymbol{w}$ is given by $\boldsymbol{w}=K \dot{q}$, where the fixture stiffness matrix $K$ is assumed positive definite. The worst case deflection of $\mathcal{B}$ is characterized as its maximal displacement in response to all wrenches in a frame-invariant unit wrench ball. This notion is precisely made in the following definition.

Definition 4: Let a fixture arrangement of an object $\mathcal{B}$ have a stiffness matrix $K$. The deflection quality measure of the fixture is given by $\Delta=\max _{\|\boldsymbol{w}\|_{\text {rms }} \leqslant 1}\left\{\|\mathrm{C} \boldsymbol{w}\|_{\mathrm{p}}\right\}$, where $C=K^{-1}$ and $\|\cdot\|_{p}$ is rms or max velocity norm. The reciprocal deflection quality measure is given by $Q=\min _{\|\dot{q}\|_{\mathrm{p}} \geqslant 1}\left\{\||\mathrm{~K} \dot{q}|\|_{\text {rms }}\right\}$, where $\|\cdot\|_{p}$ is rms or max velocity norm.

Note that $\Delta$ is frame invariant, since it is defined in terms of frame-invariant velocity and wrench norms. Note, too, that 
the velocity and wrench balls used in the definition are not Euclidean balls. These balls are frame invariant in the sense that their boundary points always have unit norm, regardless of the reference frames being used. The reciprocal quality measure $Q$ quantifies the weakest reaction wrench generated by the contacts in response to a frame-invariant collection of object displacements. It is shown below that $Q$ is indeed the reciprocal of $\Delta$. Hence, one can compute an optimal fixture arrangement either by minimizing $\Delta$ or maximizing $Q$ over the possible contacts.

In terms of applications, suppose the deflection of $\mathcal{B}$ must not exceed a tolerance $\epsilon$ which is measured in terms of a velocity norm. Then, $\Delta$ has three interesting applications corresponding to this tolerance. First, let a set of external loads vary in a wrench ball $W=\left\{\alpha \boldsymbol{w}: \boldsymbol{w} \in W_{0}, 0 \leqslant \alpha \leqslant \alpha_{\max }\right\}$, where $W_{0}$ is the unit wrench ball. Then, a candidate fixture is valid if $\Delta$ satisfies the inequality $\Delta \leqslant \epsilon / \alpha_{\max }$. Second, $\alpha_{\max }$ can be regarded as an unknown parameter representing the maximal load capacity that can be safely applied to a fixtured object. The tolerance requirement implies the inequality $\alpha \max _{\boldsymbol{w} \in \mathrm{W}_{0}}\left\{\|\mathrm{C} \boldsymbol{w}\|_{\mathrm{p}}\right\} \leqslant \epsilon$, which determines the maximal load capacity as $\alpha_{\max }=\epsilon / \Delta$. Third, the quality measure is useful for fixture planning. When a set of candidate fixel positions is parameterized by $s$, the deflection quality measure becomes a parametrized function $\Delta(s)$. The fixture planning task becomes an optimization problem of $\Delta(s)$ subject to $\epsilon$-tolerance constraints. This approach is discussed below in the context of optimal fixtures of polygonal objects.

We now turn to the computation of the deflection quality measure. First, we compute the quality measure using the rms velocity norm. This quality measure, denoted as $\Delta_{\mathrm{rms}}$, indicates $\mathcal{B}$ 's worst case average deflection over a unit wrench ball. In the following, $\lambda_{\min }(A)$ and $\lambda_{\max }(A)$ denote the smallest and largest eigenvalues of a symmetric matrix $A$.

Lemma 4: Let $M$ be the weighting matrix associated with the rms norm. The deflection quality measure for the rms velocity norm and rms wrench norm is

$$
\begin{aligned}
\Delta_{\mathrm{rms}} & =\lambda_{\max }\left(M^{\frac{1}{2}} C M^{\frac{1}{2}}\right) \text { where } \\
C & =K^{-1}\{\text { is the fixture compliance matrix. }
\end{aligned}
$$

The reciprocal deflection quality measure is given by

$$
Q_{\mathrm{rms}}=\frac{1}{\Delta_{\mathrm{rms}}}=\lambda_{\min }\left(M^{-\frac{1}{2}} K M^{-\frac{1}{2}}\right)
$$

where $K$ is the fixture stiffness matrix.

Proof: The rms unit wrench ball can be written as $\{\boldsymbol{w}$ : $\left.\|\boldsymbol{w}\|_{\mathrm{rms}} \leqslant 1\right\}=\{x:\|x\| \leqslant 1\}$, where $x=M^{-1 / 2} \boldsymbol{w}$ and $\|\cdot\|$ is the usual Euclidean norm. Replacing $\boldsymbol{w}$ with $x$ in the definition of $\Delta_{\text {rms }}$, and using the identity $\left(\max \left\{\mathrm{a}^{1 / 2}(\mathrm{r})\right\}\right)^{2}=$ $\max \{\mathrm{a}(\mathrm{r})\}$, gives

$$
\begin{aligned}
\Delta_{\mathrm{rms}}^{2} & =\max _{\|\mathrm{x}\| \leqslant 1}\left\{\mathrm{x}^{\mathrm{T}} \mathrm{M}^{\frac{1}{2}} \mathrm{CMCM}^{\frac{1}{2}} \mathrm{x}\right\} \\
& =\max _{\|\mathrm{x}\| \leqslant 1}\left\{\mathrm{x}^{\mathrm{T}}\left(\mathrm{M}^{\frac{1}{2}} \mathrm{CM}^{\frac{1}{2}}\right)^{\mathrm{T}} \mathrm{M}^{\frac{1}{2}} \mathrm{CM}^{\frac{1}{2}} \mathrm{x}\right\} \\
& =\lambda_{\max }^{2}\left(M^{\frac{1}{2}} C M^{\frac{1}{2}}\right) .
\end{aligned}
$$

Next, consider the reciprocal quality measure $Q_{\mathrm{rms}}=$ $\min _{\|\dot{q}\|_{\mathrm{rms}} \geqslant 1}\left\{\|\mid K \dot{q}\|_{\mathrm{rms}}\right\}$. We wish to show that $Q_{\mathrm{rms}}=$ $1 / \Delta_{\mathrm{rms}}$. The rms velocity norm can be written as $\|\dot{q}\|_{\mathrm{rms}}=\|y\|$ where $y=M^{1 / 2} \dot{q}$ and $\|\cdot\|$ are the usual Euclidean norm. Replacing $\dot{q}$ with $y$ and using the identity $\left(\min \left\{a^{1 / 2}(r)\right\}\right)^{2}=$ $\min \{a(r)\}$ gives

$$
\begin{aligned}
& \left(\min _{\|\dot{q}\|_{\mathrm{rms}} \geqslant 1}\left\{\|\mid K \dot{q}\|_{\mathrm{rms}}\right\}\right)^{2} \\
& =\min _{\|y\| \geqslant 1}\left\{y^{T} M^{-\frac{1}{2}} K^{T} M^{-1} K M^{-\frac{1}{2}} y\right\} \\
& =\min _{\|y\| \geqslant 1}\left\{y^{T}\left(M^{-\frac{1}{2}} K M^{-\frac{1}{2}}\right)^{T} M^{-\frac{1}{2}} K M^{-\frac{1}{2}} y\right\} \\
& =\lambda_{\min }^{2}\left(M^{-\frac{1}{2}} K M^{-\frac{1}{2}}\right) .
\end{aligned}
$$

Since $\left(M^{1 / 2} C M^{1 / 2}\right)^{-1}=M^{-1 / 2} K M^{-1 / 2}$, we have that $1 / \Delta_{\mathrm{rms}}=1 / \lambda_{\max }\left(M^{1 / 2} C M^{1 / 2}\right)=\lambda_{\min }\left(M^{-1 / 2} K M^{-1 / 2}\right)$. Thus, $Q_{\mathrm{rms}}=1 / \Delta_{\mathrm{rms}}$.

Next, we compute the deflection quality measure using the max velocity norm. This quality measure, denoted $\Delta_{\max }$, indicates $\mathcal{B}$ 's worst case pointwise deflection over a unit wrench ball. We restrict our attention to the following discretized version of $\Delta_{\max }$. Suppose $\mathcal{B}$ possesses distinguished points $r_{1}, \ldots, r_{m}$ which are critical for precision monitoring. Let the weighting function have discrete weights $\nu_{1}, \ldots, \nu_{m}$ at these points, such that $\sum_{i=1}^{m} \nu_{i}=1$. The following lemma asserts that the discretized $\Delta_{\max }$ can be efficiently computed as a collection of $m$ eigenvalue problems.

Lemma 5: Let $\mathcal{B}$ have distinguished points $r_{1}, \ldots, r_{m}$ with discrete weights $\nu_{1}, \ldots, \nu_{m}$. The deflection quality measure for the max velocity norm and rms wrench norm is

$$
\Delta_{\max }=\left(\max _{1 \leqslant \mathrm{i} \leqslant \mathrm{m}}\left\{\lambda_{\max }\left(\mathrm{M}^{\frac{1}{2}} \mathrm{C}^{\mathrm{T}} \mathcal{R} \mathrm{N}_{\mathrm{i}} \mathcal{R}^{\mathrm{T}} \mathrm{CM}^{\frac{1}{2}}\right)\right\}\right)^{\frac{1}{2}}
$$

where $C=K^{-1}, \mathcal{R}=\operatorname{diag}(R, R), M=$ $\sum_{i=1}^{m} \nu_{i}\left(\begin{array}{cc}I & \widehat{r}_{i}^{T} \\ \widehat{r}_{i} & -\widehat{r}^{2}\end{array}\right)$, and $N_{i}=\nu_{i}^{2}\left(\begin{array}{cc}I & \widehat{r}_{i}^{T} \\ \widehat{r}_{i} & -\widehat{r}^{2}\end{array}\right)$ for $i=1, \ldots, m$. Moreover, the reciprocal deflection quality measure is given by $Q_{\max }=1 / \Delta_{\max }$.

The lemma is proved in Appendix A. We conclude with an example that describes how the deflection quality measure varies over a parametrized family of 2-D frictional fixtures. It is worth noting that the quality measure holds for 2-D as well as 3-D fixtures. However, in terms of concrete examples and computations this paper focuses on 2-D fixtures.

Example 1: Consider an object $\mathcal{B}$, which is a regular pentagon with edge length $a$ (Fig. 1). The object is fixtured by three frictional quasirigid fixels using the following family of candidate fixtures [Fig. 1(a)]. In each fixture arrangement, one fixel is fixed at the midpoint of the edge $C D$. The other two fixels lie on the edges $A B$ and $A E$ at a parametrized distance $s a$ from the vertex $A$, where $0 \leqslant s \leqslant 1$. In order to compute the stiffness matrix of these frictional fixtures, we use the stiffness matrix formula ${ }^{5}$ given in [8] $K=\sum_{i=1}^{3}\left(k_{n_{i}} \phi_{i} \phi_{i}^{T}+k_{t_{i}} \psi_{i} \psi_{i}^{T}\right)$. In this formula, $k_{n_{i}}$ and $k_{t_{i}}$ are material elasticity constants along the normal and tangent directions. In addition, $\phi_{i}$ and $\psi_{i}$ are the 3 $\times 1$ vectors $\phi_{i}=\left(n_{i}, s_{n_{i}}\right)$ and $\psi_{i}=\left(t_{i}, s_{t_{i}}\right)$, where $n_{i}$ and $t_{i}$ are the unit normal and tangent vectors at the $i$ th contact, while $s_{n_{i}}$ and $s_{t_{i}}$ are the moments of $n_{i}$ and $t_{i}$ with respect to the origin. Since the deflection quality measure is frame invariant, we may choose coincident world and object frames, with their origin at $\mathcal{B}$ 's center of symmetry and their $x$-axis parallel to the

\footnotetext{
${ }^{5}$ See [40] for a detailed modeling of frictional quasirigid contacts.
} 


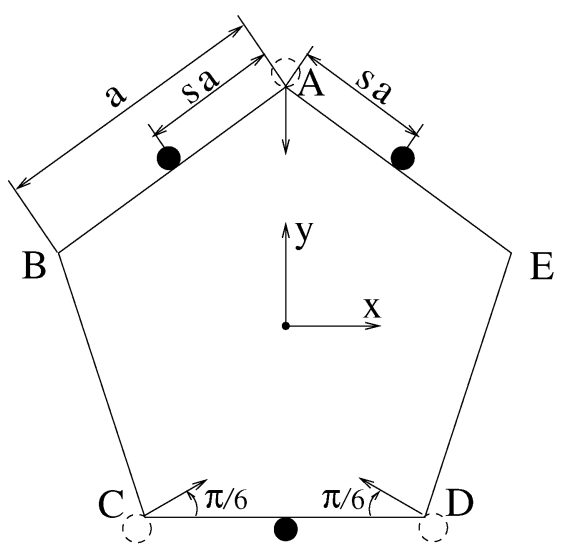

(a)

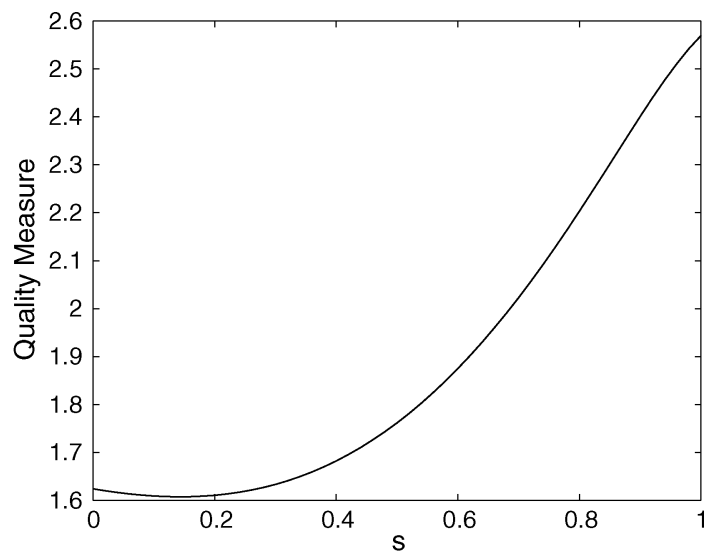

(b)

Fig. 1. (a) Three-fixel frictional fixture of a pentagon. (b) Variation of $Q_{\mathrm{rms}}=1 / \Delta_{\mathrm{rms}}$ as a function of $s$.

edge $C D$. Assuming for simplicity that $k_{n_{i}}=k_{t_{i}}=k$ (typically $k_{t_{i}}$ is smaller than $k_{n_{i}}$ ), the parametrized stiffness matrix for this fixture is

$K=k\left(\begin{array}{ccc}3 & 0 & a(1.18 s-1.01) \\ 0 & 3 & 0 \\ a(1.18 s-1.01) & 0 & a^{2}\left(2 s^{2}-2 s+1.92\right)\end{array}\right)$ where $0 \leqslant s \leqslant 1$.

We wish to examine the variation of the reciprocal quality measure $Q_{\mathrm{rms}}$ as a function of $s$. Let us choose a discrete weighting function $\nu(r)=(1 / 5) \sum_{i=1}^{5} \delta\left(r-r_{i}\right)$, where $r_{1}, \ldots, r_{5}$ are $\mathcal{B}$ 's vertices. It can be verified that the weighting matrix in this case is simply $M=\operatorname{diag}\left(1,1, \rho^{2}\right)$, where $\rho=(1 / 2) a \sec (\pi / 5)$ is the distance from $\mathcal{B}$ 's center of symmetry to the vertices. Using Lemma 4 , the reciprocal quality measure is given in terms of $\widetilde{K}=M^{-1 / 2} K M^{-1 / 2}$ by

$$
\begin{aligned}
Q_{\mathrm{rms}} & =\lambda_{\min }(\widetilde{K}) \text { with } \\
\widetilde{K} & =k\left(\begin{array}{ccc}
3 & 0 & 1.38 s-1.19 \\
0 & 3 & 0 \\
1.38 s-1.19 & 0 & 2.76 s^{2}-2.76 s+2.65
\end{array}\right) .
\end{aligned}
$$

The variation of $Q_{\mathrm{rms}}$ as a function of $s$ is plotted in Fig. 1(b). It can be seen that $Q_{\mathrm{rms}}$ is initially insensitive to the fixel locations. As $s$ further increases, the two upper fixels spread apart and $Q_{\mathrm{rms}}$ increases monotonically at a significant rate. This agrees with our intuition that the object deflection $\Delta_{\mathrm{rms}}=$ $1 / Q_{\mathrm{rms}}$ should decrease in a spread-apart fixel arrangement. A more detailed characterization of minimum-deflection fixtures is provided in the next section.

\section{Minimum Deflection Fixtures of Polygons}

In this section, we focus on the task of computing globally optimal fixtures of 2-D polygonal objects, using three or four fixels. We describe techniques for computing optimal fixtures of polygonal objects, where optimality is determined by the rms deflection quality measure. In doing so, we also wish to gain qualitative understanding of the optimal contact arrangements. The computation is based on the reciprocal quality measure $Q_{\mathrm{rms}}=\lambda_{\min }\left(M^{-1 / 2} K M^{-1 / 2}\right)$, where $M$ is the object's weighting matrix and $K$ is the fixture stiffness matrix. We first provide a concise formula for the key matrix
$\widetilde{K}=M^{-1 / 2} K M^{-1 / 2}$, called the scaled stiffness matrix, then discuss optimal fixturing using three and four fixels.

\section{A. Formula for the Scaled Stiffness Matrix}

The scaled stiffness matrix formula relies on the following terminology. The weighting matrix $M$ is given in the 2-D case by $M=\int_{\mathcal{B}} \nu(r)\left(\begin{array}{cc}I & -J r \\ (-J r)^{T} & \|r\|^{2}\end{array}\right) d r$, where $J=\left(\begin{array}{cc}0 & 1 \\ -1 & 0\end{array}\right)$. Since the deflection quality measure is frame invariant, we may choose the world and body frames $\mathcal{F}_{W}$ and $\mathcal{F}_{B}$ to be coincident. When the origin of these frames is selected at $\mathcal{B}$ 's centroid with respect to the weighting function $\nu$, the weighing matrix becomes diagonal $M=\operatorname{diag}\left(1,1, \rho^{2}\right)$, where $\rho=\left(\int_{\mathcal{B}} \nu(r)\|r\|^{2} d r\right)^{1 / 2}$. Recall that $\rho$ is $\mathcal{B}$ 's radius of gyration when $\nu$ is mass density. Here, $\rho$ is a kinematic quantity called the radius of $\mathcal{B}$ with respect to the weighting function $\nu$. Next, we introduce several quantities associated with the stiffness matrix. Let $K=\left(\begin{array}{ll}K_{11} & K_{12} \\ K_{12}^{T} & K_{22}\end{array}\right)$, where $K_{11}, K_{12}$, and $K_{22}$ are $2 \times 2,2 \times 1$, and $1 \times 1$. The stiffness matrix is associated with a special point in the plane, called center of compliance and denoted $p_{c}$, whose location is given by $p_{c}=J K_{11}^{-1} K_{12}$. When $\mathcal{F}_{W}$ and $\mathcal{F}_{B}$ are selected at $p_{c}$ with suitable orientation, the stiffness matrix becomes diagonal $K=\operatorname{diag}\left(\sigma_{1}, \sigma_{2}, \mu\right)$. The scalars $\sigma_{1}$ and $\sigma_{2}$, called the translational stiffnesses of $K$, are the eigenvalues of $K_{11}$. The scalar $\mu$, called the rotational stiffness of $K$, is given by $\mu=K_{22}-K_{12}^{T} K_{11}^{-11} K_{12}$. The following lemma specifies a formula for the scaled stiffness matrix in terms of these parameters.

Lemma 6: Let a fixture arrangement of a planar object $\mathcal{B}$ have a stiffness matrix $K$. The scaled stiffness matrix for frames $\mathcal{F}_{W}$ and $\mathcal{F}_{B}$ coincident at $\mathcal{B}$ 's centroid is given by

$$
\widetilde{K}=\left(\begin{array}{ccc}
\sigma_{1} & 0 & -\sigma_{1} \frac{\eta}{\rho} \\
0 & \sigma_{2} & \sigma_{2} \frac{\xi}{\rho} \\
-\sigma_{1} \frac{\eta}{\rho} & \sigma_{2} \frac{\xi}{\rho} & \frac{\mu}{\rho^{2}}+\sigma_{2}\left(\frac{\xi}{\rho}\right)^{2}+\sigma_{1}\left(\frac{\eta}{\rho}\right)^{2}
\end{array}\right)
$$

where $\sigma_{1}, \sigma_{2}$, and $\mu$ are the translational and rotational stiffnesses of $K, p_{c}=(\xi, \eta)$ is the center of compliance, and $\rho$ is the radius of $\mathcal{B}$ associated with the weighting function $\nu$. 
The lemma is obtained in two steps. First, one applies the transformation rule (2) to obtain the formula $K=T^{T} \operatorname{diag}\left(\sigma_{1}, \sigma_{2}, \mu\right) T$, where $T$ is the transformation from $\mathcal{B}$ 's center of compliance to its centroid. Then, one computes the scaled stiffness matrix $\widetilde{K}=$ $M^{-1 / 2} K M^{-1 / 2}=\operatorname{diag}(1,1,1 / \rho) K \operatorname{diag}(1,1,1 / \rho)$. As a shorthand notation, we define $\widetilde{\xi}=\xi / \rho, \widetilde{\eta}=\eta / \rho$, and $\widetilde{\mu}=\mu / \rho^{2}$, so that the scaled stiffness matrix becomes

$$
\widetilde{K}=\left(\begin{array}{ccc}
\sigma_{1} & 0 & -\sigma_{1} \widetilde{\eta} \\
0 & \sigma_{2} & \sigma_{2} \widetilde{\xi} \\
-\sigma_{1} \widetilde{\eta} & \sigma_{2} \widetilde{\xi} & \widetilde{\mu}+\sigma_{2} \widetilde{\xi}^{2}+\sigma_{1} \widetilde{\eta}^{2}
\end{array}\right) .
$$

The formula for $\widetilde{K}$ is valid for planar fixtures represented by any quasirigid compliance model, which may involve frictional contacts (Example 1). However, for illustration purposes, we make several assumptions for the rest of this section. First, we assume frictionless quasirigid contacts. This assumption is well justified in fixturing applications where friction effects are often neglected. The classical contact theory of Hertz [15] describes the nonlinear mechanics of such contacts, and we use stiffness matrices which are based on this model [27], [30]. Additionally, we assume that the fixels are disc shaped with radius $r$ and located at interior points of the object's edges.

\section{B. Minimum Deflection Fixtures Using Three Fixels}

We describe a graphical technique for computing the minimum deflection a three-contact fixture of polygonal objects. Our first task is to characterize the three-contact stable equilibrium fixtures of the given object. A three-contact equilibrium is fully characterized by the condition that the contact normals positively span the origin such that their lines intersect at a common point. We discard contact arrangements where the three contacts lie on two parallel edges of $\mathcal{B}$, since without friction, such fixtures are only neutrally stable. Thus, we focus on nonparallel edge triplets that admit three-contact equilibrium fixtures, and refer to these as admissible edge triplets. An admissible edge triplet yields a stable fixture when the stiffness matrix associated with the fixture is positive definite. The following lemma gives a formula for the stiffness matrix (a proof of the lemma can be found in [27]). In the lemma, $n_{i}$ is the $i$ th inward unit normal to an edge of $\mathcal{B}$, and $k_{i}$ is material stiffness at the $i$ th contact. The circumscribing circle of a triangle is the circle which passes through the triangle's vertices (Fig. 2).

Lemma 7: Let three disc fixels hold a polygonal object in a frictionless equilibrium fixture. The fixture's center of compliance lies at the contact normals concurrency point, and for frames $\mathcal{F}_{W}$ and $\mathcal{F}_{B}$ coincident at this point, the stiffness matrix is given by

$$
K=\operatorname{diag}\left(\sum_{i=1}^{3} k_{i} n_{i} n_{i}^{T}, \mu\right) \text { where } \mu=f_{T}(2 a \gamma+r) .
$$

In this formula, $f_{T}=\sum_{i=1}^{3} f_{i_{0}}$, where $f_{i_{0}}$ is the $i^{\text {th }}$ preloading fixel force in the absence of an external wrench; $a$ is the radius of the circumscribing circle of the edge triplet containing the contacts; $\gamma=\left(\prod_{i=1}^{3} \sin \alpha_{i}\right) /\left(\sum_{i=1}^{3} \sin \alpha_{i}\right)$ is determined from the triangle's three interior angles, denoted $\alpha_{i}$ ( $i=1,2,3)$; and $r$ is the fixels' radius.

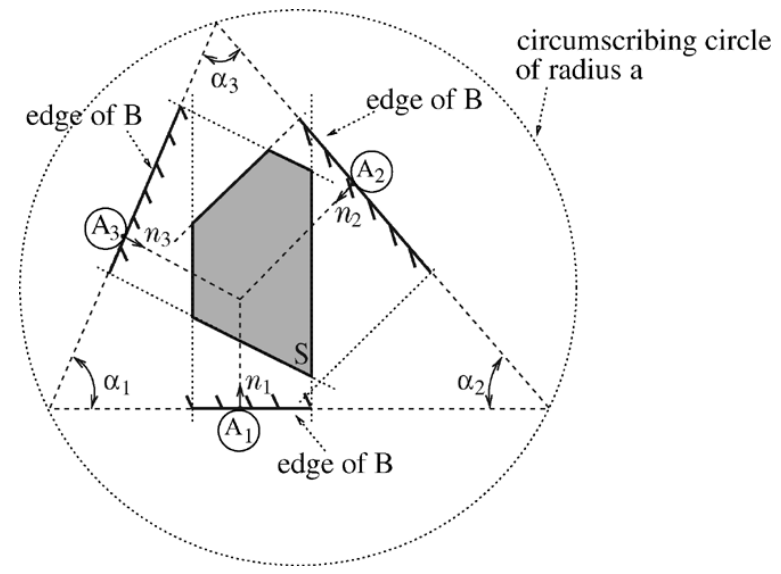

Fig. 2. Three fixels $\mathcal{A}_{1}, \mathcal{A}_{2}$, and $\mathcal{A}_{3}$ contacting a particular edge triplet.

Note that the two eigenvalues of the matrix $K_{11}=$ $\sum_{i=1}^{3} k_{i} n_{i} n_{i}^{T}$ are the translational stiffnesses $\sigma_{1}$ and $\sigma_{2}$, while $\mu$ is the rotational stiffness of the fixture. Our next task is to verify under what conditions $K$ is positive definite. First, $K_{11}$ is automatically positive definite on an admissible edge triplet. The rotational stiffness is given by $\mu=f_{T}(2 a \gamma+r)$. The parameters $a$ and $r$ are positive constants while $\gamma$ is a positive constant in compressive fixtures where the fixels push toward the concurrency point. ${ }^{6}$ Assuming compressive fixtures, $\mu$ is positive when $f_{T}$ is strictly positive. Since $f_{T}$ is the sum of the preloading forces, the requirement $f_{T}>0$ implies that the fixels must be preloaded for stability. We therefore assume that $f_{T}$ has a fixed positive value for all possible fixel placements. This is a reasonable assumption since, in practice, one often wishes to compare different fixtures having a common preloading level $f_{T}$, which is predetermined by task specifications and material strength limits. Under the assumption of a fixed $f_{T}$, the rotational stiffness $\mu$ is a positive constant on each edge triplet. Hence, all preloaded compressive equilibrium fixtures on admissible edge triplets are automatically stable.

In order to obtain a simple formula for the deflection quality measure, we invoke in Appendix B, a basic characteristic of quasirigid bodies. These bodies experience contact deformations whose dimension is much smaller than the bodies' characteristic dimension. Based on this fact, it is shown in the Appendix that $\widetilde{\mu} \ll \sigma_{1}, \sigma_{2}$ in formula (7) for $\widetilde{K}$. Since $Q_{\mathrm{rms}}=\lambda_{\min }(\widetilde{K})$, the latter inequality implies the following proposition (Appendix B).

Proposition 8: Let a polygonal object be stably fixtured by three frictionless quasirigid contacts. The reciprocal rms deflection quality measure is approximately given by

$$
Q_{\mathrm{rms}}=\frac{\mu}{\rho^{2}+z^{2}}
$$

where $\mu$ is the fixture's rotational stiffness, $\rho$ is $\mathcal{B}$ 's radius with respect to the weighting function $\nu$, and $z$ is the distance between the concurrency point of the contact normals and $\mathcal{B}$ 's centroid with respect to $\nu$.

We can now analyze how $Q_{\mathrm{rms}}$ changes as the contacts vary along a particular edge triplet. The equilibrium arrangements

\footnotetext{
${ }^{6} \gamma$ is negative in expansive fixtures where the fixels push away from the concurrency point.
} 


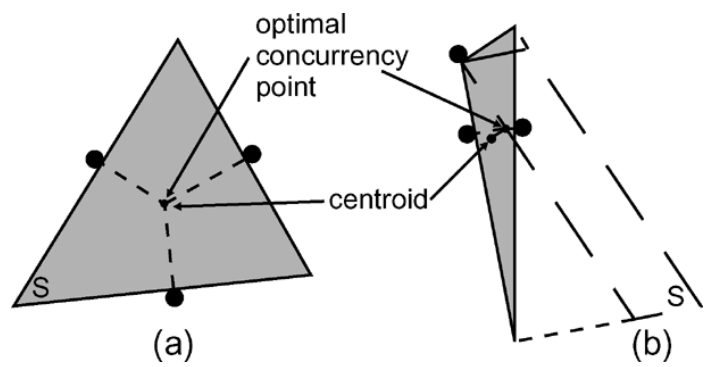

Fig. 3. Optimal three-contact fixtures of two triangular objects (shown shaded). For a uniform weighting function $\mathcal{B}$ 's centroid lies (a) inside $S$ and (b) outside $S$ (fixels are black discs).

can be parametrized by the concurrency point of the contact normals, denoted as $p$. This point lies inside a convex polygonal region, denoted as $S$, formed by intersecting three strips whose bounding lines are perpendicular to the three edges and pass through their endpoints (Fig. 2). Since $f_{T}$ is fixed, the rotational stiffness $\mu$ is constant regardless of the location of $p$. The radius $\rho$ associated with the weighting function is also constant. Hence, the only variable that changes with $p$ is the distance $z$. It follows that $Q_{\mathrm{rms}}$ is maximal (and, hence, $\Delta_{\mathrm{rms}}$ is minimal) on a given edge triplet when $z$ is minimal. The problem of finding the minimum deflection fixture on a given edge triplet is thus equivalent to the minimization of $z(p)$ over $p \in S$.

The above discussion leads to the following graphical technique for computing the optimal fixture on a given edge triplet. (The optimization over different edge triplets is considered below.) First, find the region $S$ as shown in Fig. 2. Second, find the centroid of $\mathcal{B}$ with respect to the weighting function $\nu$. If the centroid lies in $S$, it is the optimal concurrency point of the contact normals. Otherwise, the centroid lies outside $S$. Since $S$ is a polygonal region, the optimal concurrency point is the closest point in $S$ to $\mathcal{B}$ 's centroid-this point either lies on an edge of $S$ or is a vertex of $S$. Finally, knowledge of the optimal concurrency point determines the optimal fixel locations. The graphical technique is illustrated with the following example.

Example 2: The minimum deflection fixture of a triangle or a particular edge triplet can be graphically determined as shown in Fig. 3. First, the region $S$ of possible concurrency point positions must be determined. When $\mathcal{B}$ 's centroid lies inside $S$, the optimal concurrency point coincides with $\mathcal{B}$ 's centroid as shown in Fig. 3(a). When $\mathcal{B}$ 's centroid lies outside $S$, a perpendicular line can be drawn through the centroid to the closest boundary edge of $S$. The resulting intersection gives the optimal concurrency point, as shown in Fig. 3(b).

Our last task is to characterize the globally optimal fixture over all admissible edge triplets. While $\mu$ is constant on a particular edge triplet, it attains different values on different edge triplets. When $f_{T}$ is fixed, $\mu=f_{T}(2 a \gamma+r)$ is influenced by the shape and size of the triangle determined by each edge triplet. The size parameter $a$ (the radius of the edge-triplet's circumscribing circle) and the shape parameter $\gamma$ (determined by the edge-triplet's interior angles) are both preferred to be large for achieving minimum deflection. Therefore, in the globally optimal fixture, the fixels are spread apart, and the edges in the triplet are oriented evenly. Lastly, $Q_{\mathrm{rms}}=\mu /\left(\rho^{2}+z^{2}\right)$. Hence, the distance $z$ between $\mathcal{B}$ 's centroid and the concurrency point of

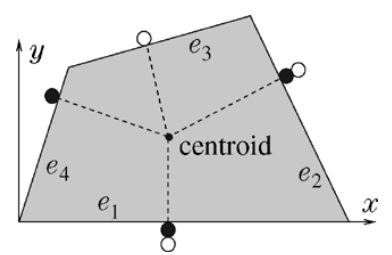

Fig. 4. Optimal three-contact minimum deflection fixture is on edge triplet $\left(e_{1}, e_{2}, e_{4}\right)$.

the contact normals must be small. However, the optimal fixture is determined by a combined influence of the above parameters, as illustrated with the following example.

Example 3: Consider the minimum deflection fixture of the quadrilateral object shown in Fig. 4, using the limiting case of zero fixel radii. The vertices of $\mathcal{B}$ have coordinates $(0,0)$, $(1,0),(0.7,0.6),(0.15,0.45)$. We choose a weighting function $\nu(r)=(1 / 4) \delta\left(r-r_{i}\right)$, where $r_{i}$ are the object's vertices. Under this choice, $\mathcal{B}$ 's centroid has coordinates $(0.4625,0.2625)$, while $\mathcal{B}$ 's radius is $\rho=0.4857$. The object has only two admissible edge triplets $\left(e_{1}, e_{2}, e_{3}\right)$ and $\left(e_{1}, e_{2}, e_{4}\right)$, and the minimum deflection fixture for each triplet is shown in Fig. 4. The optimal concurrency point for both triplets coincides with $\mathcal{B}$ 's centroid. However, the two edge triplets have different shapes and sizes. For the triplet $\left(e_{1}, e_{2}, e_{3}\right), a \gamma=0.1376$ and, therefore, $Q_{\mathrm{rms}}=1.1665 f_{T}$. For the triplet $\left(e_{1}, e_{2}, e_{4}\right), a \gamma=0.1664$ and, therefore, $Q_{\mathrm{rms}}=1.4105 f_{T}$. It follows that the optimal minimum-deflection fixture is the one on $\left(e_{1}, e_{2}, e_{4}\right)$.

\section{Minimum Deflection Fixtures Using Four Fixels}

The usage of four rather than three fixels provides a more robust immobilization in which the contact normals determine the fixture's translational as well as rotational stiffness. We describe a technique for computing the minimum deflection four-contact fixture of a polygonal object. Here, too, our first task is to characterize the four-contact stable equilibrium fixtures. The four fixels can be placed on any edge triplet or quadruplet of $\mathcal{B}$, and we first focus on a particular edge combination. The four-contact arrangements on a given edge combination are parametrized as follows. Let $O$ be the origin of $\mathcal{F}_{B}$ and let $e_{i}$ be the edge containing the $i$ th contact. The $i$ th contact is parametrized by its signed distance, denoted $s_{i}$, measured along $e_{i}$ from the point where $e_{i}$ or its extension perpendicularly intersects a line passing through $O$. The vector $\boldsymbol{s}=\left(s_{1}, s_{2}, s_{3}, s_{4}\right)$ specifies a particular contact configuration, and the collection of all contact configurations on a given edge combination forms a four-dimensional (4-D) cube denoted $\mathcal{P}$.

Next, we characterize the four-contact equilibrium configurations in $\mathcal{P}$. First, note that $s_{i}$ can be interpreted as the torque generated by a unit force $n_{i}$ acting on $\mathcal{B}$ at the $i$ th contact. The wrench generated by the unit force $n_{i}$ is therefore $\boldsymbol{w}_{i}=\left(n_{i}, s_{i}\right)$. A four-contact equilibrium fixture is characterized by the condition that a positive linear combination of the wrenches $\boldsymbol{w}_{1}, \ldots, \boldsymbol{w}_{4}$ spans the zero wrench. This condition is valid on the following subset of $\mathcal{P}$. Let the functions $d_{i}(\boldsymbol{s})=\operatorname{det}\left(\left[\boldsymbol{w}_{i+1} \boldsymbol{w}_{i+2} \boldsymbol{w}_{i+3}\right]\right)(\bmod 4)$ be called the determinant functions associated with the given edge combination. Then, we have the following lemma. 
Lemma 9 ([27]): Let a planar object be held by four fixels at a contact configuration $\boldsymbol{s}$. A necessary and sufficient condition for $\boldsymbol{s}$ to be an equilibrium fixture is that $d_{1}(\boldsymbol{s}),-d_{2}(\boldsymbol{s}), d_{3}(\boldsymbol{s})$, and $-d_{4}(\boldsymbol{s})$ are all nonzero and have the same sign.

The lemma implies that the collection of four-contact equilibrium fixtures on a particular edge combination is a disjoint union of two 4-D sets $\mathcal{T}_{1} \cup \mathcal{T}_{2}$, where

$$
\begin{aligned}
& \mathcal{T}_{1}=\mathcal{P} \cap\left\{\boldsymbol{s} \in \mathbb{R}^{4}: d_{1}(\boldsymbol{s}),-d_{2}(\boldsymbol{s}), d_{3}(\boldsymbol{s}),-d_{4}(\boldsymbol{s})<0\right\} \\
& \mathcal{T}_{2}=\mathcal{P} \cap\left\{\boldsymbol{s} \in \mathbb{R}^{4}: d_{1}(\boldsymbol{s}),-d_{2}(\boldsymbol{s}), d_{3}(\boldsymbol{s}),-d_{4}(\boldsymbol{s})>0\right\} .
\end{aligned}
$$

For a polygonal object, each $d_{i}$ is linear in $\boldsymbol{s}$. Hence, $\mathcal{T}_{1}$ and $\mathcal{T}_{2}$ are bounded convex polytopes in $\mathbb{R}^{4}$. Thus, for a given edge combination, one may separately compute the optimal fixture on $\mathcal{T}_{1}$ and $\mathcal{T}_{2}$, then select the best fixture among the two polytopes. Our next task is to characterize the stable four-contact fixtures in $\mathcal{T}_{1}$ and $\mathcal{T}_{2}$. The following formula specifies the stiffness matrix for a four-contact fixture of a polygonal object.

Lemma 10 ([27]): Let four disc fixels hold a polygonal object in a frictionless equilibrium fixture at a contact configuration $\boldsymbol{s}=\left(s_{1}, s_{2}, s_{3}, s_{4}\right)$. For coincident frames $\mathcal{F}_{W}$ and $\mathcal{F}_{B}$ (with origin anywhere in the plane), the fixture stiffness matrix is given by

$$
K(\boldsymbol{s})=\sum_{i=1}^{4} k_{i} \boldsymbol{w}_{i} \boldsymbol{w}_{i}^{T}=\sum_{i=1}^{4} k_{i}\left(\begin{array}{cc}
n_{i} n_{i}^{T} & s_{i} n_{i} \\
s_{i} n_{i}^{T} & s_{i}^{2}
\end{array}\right)
$$

where $k_{i}$ is the $i$ th contact stiffness, and $\boldsymbol{w}_{i}=\left(n_{i}, s_{i}\right)$ is the wrench generated by an inward unit normal force at the $i$ th contact.

One can verify that $K$ is positive definite in the generic case where the $3 \times 4$ matrix $\left[\sqrt{k_{1}} \boldsymbol{w}_{1} \cdots \sqrt{k_{4}} \boldsymbol{w}_{4}\right]$ has full rank. Hence, all generic four-contact equilibrium fixtures are automatically stable. Note that in contrast with the three-contact stiffness matrix, the fixels' radius does not appear in (10). Formula (10) is, in fact, a simplification of an exact formula which contains a curvature-dependent term [30]. However, the latter term is typically much smaller than the term appearing in (10) and does not affect fixture stability.

Having obtained a formula for $K$, we proceed to derive expressions for the translational and rotational stiffnesses of $K$ in terms of $\boldsymbol{s}$. These parameters appear in the formula for the scaled stiffness matrix $\widetilde{K}$, whose minimal eigenvalue is $Q_{\text {rms }}$. The translational stiffnesses $\sigma_{1}$ and $\sigma_{2}$ are the eigenvalues of the submatrix $K_{11}=\sum_{i=1}^{4} k_{i} n_{i} n_{i}^{T}$. The rotational stiffness is given by $\mu=K_{22}-K_{12}^{T} K_{11}^{-1} K_{12}$. Substitution of these submatrices according to (10) gives

$\mu(\boldsymbol{s})=\sum_{i=1}^{4} k_{i} s_{i}^{2}-\left(\sum_{i=1}^{4} k_{i} s_{i} n_{i}^{T}\right)-\left(\sum_{i=1}^{4} k_{i} n_{i} n_{i}^{T}\right) \sum_{i=1}^{4} k_{i} s_{i} n_{i}$.

It can be verified that for polygonal objects and disc fixels, the contact stiffnesses $k_{i}$ remain approximately constant as the fixels move along a particular edge combination. Hence, we make the simplifying assumption that the $k_{i}$ 's are constant on each edge combination. It follows that $\sigma_{1}$ and $\sigma_{2}$ are constant on a given edge combination. However, the rotational stiffness $\mu(\boldsymbol{s})$ is a non-negative quadratic function of $\boldsymbol{s}$.
Before proceeding with the computation of the optimal four-contact fixture, let us pause to discuss some of its qualitative properties. The discussion is based on the following lemma (Appendix B).

Lemma 11: Let a polygonal object $\mathcal{B}$ be held by four fixels. Let $\rho$ be $\mathcal{B}$ 's radius with respect to the weighting function $\nu$. As the contacts vary along a particular edge combination, $Q_{\mathrm{rms}}$ satisfies the lower bound

$$
\begin{aligned}
Q_{\mathrm{rms}}(\boldsymbol{s}) \geqslant \min \left\{\sigma_{1},\right. & \sigma_{2}, \frac{1}{2}\left(\sigma \widetilde{z}^{2}(\boldsymbol{s})+\widetilde{\mu}(\boldsymbol{s})+\sigma\right. \\
& \left.\left.-\sqrt{\left(\sigma \widetilde{z}^{2}(\boldsymbol{s})+\widetilde{\mu}(\boldsymbol{s})+\sigma\right)^{2}-4 \sigma \widetilde{\mu}(\boldsymbol{s})}\right)\right\}
\end{aligned}
$$

where $\sigma=\min \left\{\sigma_{1}, \sigma_{2}\right\}, \widetilde{\mu}=\mu(\boldsymbol{s}) / \rho^{2}$, and $\widetilde{z}=z(\boldsymbol{s}) / \rho$, where $z(\boldsymbol{s})$ is the distance between the fixture's center of compliance and $\mathcal{B}$ 's centroid. Moreover, the lower bound is monotonically increasing in $\mu(\boldsymbol{s})$ and monotonically decreasing in $z(\boldsymbol{s})$.

The lemma implies the following generic properties of the minimum deflection four-contact fixture (i.e., the one which maximizes $Q_{\mathrm{rms}}$ ). First, $Q_{\mathrm{rms}} \geqslant \min \left\{\sigma_{1}, \sigma_{2}\right\}$, where $\sigma_{1}$ and $\sigma_{2}$ are the eigenvalues of $K_{11}=\sum_{i=1}^{4} k_{i} n_{i} n_{i}^{T}$. It can be verified that the eigenvalues of $K_{11}$ are the largest when the contact normals are evenly oriented. Second, the rotational stiffness $\mu(\boldsymbol{s})$ is quadratic in $\boldsymbol{s}$. Recall now that $s$ represents the moments generated by the fixels. In particular, $\mu(\boldsymbol{s})$ increases monotonically with $\|\boldsymbol{s}\|$ when the moments are measured with respect to the fixture's center of compliance. Thus, for larger values of $Q_{\mathrm{rms}}$, the fixels should spread apart with respect to the center of compliance so that the moments generated by the fixels would be as large as possible. Lastly, the lower bound on $Q_{\text {rms }}$ decreases monotonically with the distance $z$. Hence, for larger values of $Q_{\mathrm{rms}}$, this distance should be as small as possible, and most desirably should be zero. The following example illustrates some of these observations.

Example 4: Consider the fixturing of a rectangular object using the following family of four-contact arrangements (Fig. 5). Two fixels are located on the vertical edges of $\mathcal{B}$ at a signed distance $s_{1}$ from the $x$-axis, and two fixels are located on the horizontal edges of $\mathcal{B}$ at a signed distance $s_{2}$ from the $y$-axis. The center of compliance of these symmetric contact arrangements coincides with $\mathcal{B}$ 's center of symmetry. When we choose a weighting function $\nu(r)=(1 / 4) \delta\left(r-r_{j}\right)$, where $r_{j}$ are $\mathcal{B}$ 's vertices, the centroid of $\mathcal{B}$ with respect to $\nu$ also coincides with $\mathcal{B}$ 's center of symmetry. Assuming point fixels and uniform contact stiffnesses $\left(k_{i} \underset{\widetilde{K}}{ } k\right.$ for $\left.i=1, \ldots, 4\right)$, the scaled stiffness matrix takes the form $\widetilde{K}=2 k \operatorname{diag}\left(1,1,\left(s_{1}^{2}+s_{2}^{2}\right) /\left(a^{2}+b^{2}\right)\right)$. Hence, $Q_{\mathrm{rms}}=\lambda_{\min }(\widetilde{K})$ is maximal at $\left(s_{1}, s_{2}\right)=(a, b)$. The fixels in this optimal arrangement are spread apart and positioned at diagonally opposite corners of $\mathcal{B}$ (Fig. 5).

We now proceed with the computation of the optimal fourcontact fixture on a particular edge combination. This computation requires maximization of $Q_{\mathrm{rms}}$ on the convex polytopes $\mathcal{T}_{1}$ and $\mathcal{T}_{2}$. We describe a procedure for maximizing $Q_{\mathrm{rms}}$ over one of these polytopes, denoted $\mathcal{T}$. The procedure is based on an auxiliary function $\psi$, which is defined as follows. Recall that $\widetilde{\mu}$ is the normalized rotational stiffness and $(\widetilde{\xi}, \widetilde{\eta})$ are the normalized coordinates of the center of compliance. Let us define the ratio $c=\sigma_{1} / \sigma_{2}$, where $\sigma_{1}$ and $\sigma_{2}$ are the transla- 


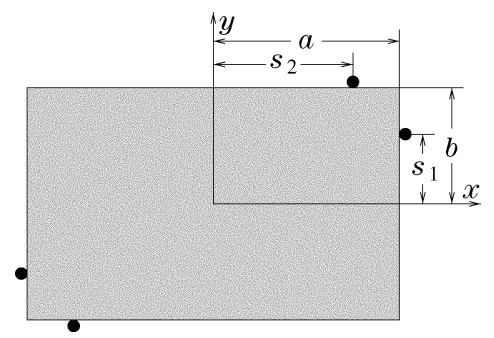

Fig. 5. Symmetric four-contact fixtures of a rectangular object.

tional stiffnesses of $K$. Then, $\psi:(0,1) \rightarrow \mathbb{R}$ is defined by $\psi(t)=\max _{\boldsymbol{s} \in \mathcal{T}}\{\phi(\mathrm{t}, \boldsymbol{s})\}$, where

$$
\begin{aligned}
\phi(t, \boldsymbol{s})=\widetilde{\mu}(\boldsymbol{s})+\left(1-\frac{1}{1-c(1-t)}\right) \sigma_{2} \widetilde{\xi}^{2}(\boldsymbol{s}) \\
\quad+\left(1-\frac{1}{t}\right) \sigma_{1} \widetilde{\eta}^{2}(\boldsymbol{s})-(1-t) \sigma_{1} .
\end{aligned}
$$

The following proposition specifies a procedure for computing the maximum of $Q_{\mathrm{rms}}$ over $\mathcal{T}$. The proposition is motivated and proved in Appendix B.

Proposition 12: The function $\psi$ has, at most, one root in the interval $(0,1)$. The maximum of $Q_{\mathrm{rms}}(\boldsymbol{s})=\lambda_{\min }(\widetilde{K}(\boldsymbol{s}))$ over $\mathcal{T}$ is determined according to the following two cases.

1) There exists $t^{*} \in(0,1)$ such that $\psi\left(t^{*}\right)=0$ if and only if $t^{*}$ satisfies the condition $\max _{\boldsymbol{s} \in \mathcal{T}}\left\{\lambda_{\min }(\widetilde{\mathrm{K}}(\mathrm{s}))\right\}=(1-$ $\left.\mathrm{t}^{*}\right) \min \left\{\sigma_{1}, \sigma_{2}\right\}$.

2) The function $\psi$ has no zero in the interval $(0,1)$ if and only if $\max _{\boldsymbol{s} \in \mathcal{T}}\left\{\lambda_{\min }(\widetilde{\mathrm{K}}(\boldsymbol{s}))\right\}=\min \left\{\sigma_{1}, \sigma_{2}\right\}$.

In order to apply the proposition, one has to compute the zero of $\psi$ in the interval $(0,1)$. Using binary search, an $\epsilon$-approximate root can be found in $\log (1 / \epsilon)$ evaluations of $\psi$. Each evaluation of $\psi$ requires a maximization of $\phi$ over $\mathcal{T}$ as described below. If no root of $\psi$ exists, one determines the optimal fixture by evaluating $\psi$ at values of $t$ that approach zero. Consider now the evaluation of $\psi$ for a fixed $t, \psi(t)=\max _{\boldsymbol{s} \in \mathcal{T}}\{\phi(\mathrm{t}, \boldsymbol{s})\}$. Since $(\widetilde{\xi}(\boldsymbol{s}), \widetilde{\eta}(\boldsymbol{s}))$ are linear and $\widetilde{\mu}(\boldsymbol{s})$ is quadratic in $\boldsymbol{s}$, the maximization of $\phi$ over $\mathcal{T}$ is an indefinite quadratic programming problem. Such problems are NP hard, and known algorithms are exponential in the number of variables. For example, [10] and [46] describe an $\epsilon$-approximate algorithm that takes $O\left(l((m(m+1) / \sqrt{\epsilon}))^{p}\right)$ steps, where $m$ is the number of variables and $p$ is the number of negative eigenvalues of the quadratic objective function (note that $p \leqslant m$ ). In this bound, $l$ is the time it takes to solve a convex quadratic optimization problem of the same size, which is polynomial in the number of linear constraints of the polytope $\mathcal{T}$. However, in our case, $m=4$ and $p \leqslant 4$. Hence, the number of steps is linear in the number of constraints, with a somewhat large constant determined by the dimension $m=4$. Given that $m$ is small, our approach provides a practical procedure for finding an $\epsilon$-approximate optimal fixture at a reasonable computational cost, despite the nonconvex and highly nonlinear nature of the optimization procedure. The procedure is illustrated with the following example.

Example 5: We apply the numerical procedure to the quadrilateral object from Example 3, with vertices at $(0,0)$, $(1,0),(0.7,0.6)$, and $(0.15,0.45)$. Using the same weighting

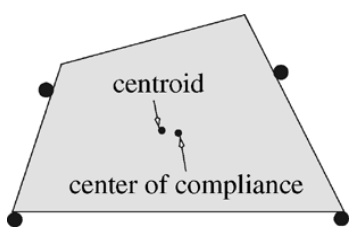

Fig. 6. Minimum deflection fixture of a quadrilateral object.

function $\nu(r)=(1 / 4) \delta\left(r-r_{j}\right)$, where $r_{j}$ are $\mathcal{B}$ 's vertices, $\mathcal{B}$ 's radius is $\rho=0.4857$ and $\mathcal{B}$ 's centroid is located at $(0.4625$, 0.2625). According to Proposition 12, the optimal fixture on each edge combination can be numerically found as an indefinite quadratic optimization problem. In our implementation, we assumed point fixels and uniform contact stiffnesses $\left(k_{i}=k\right.$ for $i=1, \ldots, 4)$. The globally optimal fixture computed over all edge triplets and quadruples of $\mathcal{B}$ is shown in Fig. 6. For this minimum deflection fixture, $\sigma_{1}=1.6838 k, \sigma_{2}=2.3162 k$, and $Q_{\mathrm{rms}}=1.6838 k=\sigma_{1}$. In contrast with the previous example, the fixels are not located at diagonally opposite corners, since such a choice would move the fixture's center of compliance away from $\mathcal{B}$ 's centroid. The precise tradeoff between such qualitative factors can only be determined by the numerical procedure. In this example, two fixels are located at the opposite corners of the bottom edge, while the other two fixels are located on the side edges just beyond the closest point to $\mathcal{B}$ 's centroid.

To summarize, the optimal three-contact fixture on an edge triplet can be graphically determined, while the optimal four-contact fixture on an edge triplet or quadruplet can be determined by a small indefinite quadratic program. In order to find the globally optimal fixture, one can repeat this computation over all edge combinations that yield feasible equilibrium grasps. The number of edge triplets and quadruplets is polynomial in the number of edges, but a more efficient combinatorial search would be required for objects having a large number of edges.

\section{CONCLUSION}

The deflection of a fixtured object has been formalized using velocity and wrench norms. These norms, in contrast to those used in previous works, are frame invariant and have interesting physical interpretations that make them suitable for practical fixture planning. Using these norms, a quality measure for compliant fixtures was defined as the norm of the object's worstcase displacement induced by a unit wrench ball. Interpreted intuitively, this quality measure gives the worst-case object deflection in response to all unit magnitude wrenches. We observed the following features of the deflection quality measure. First, the deflection quality measure is frame invariant. Second, the quality measure applies to 2-D and 3-D compliant fixtures modeled by any quasirigid compliance model and employing any number of contacts. We emphasized frictionless quasirigid models which are most relevant for fixturing applications. However, models that include friction effects can also be used. Finally, the quality measure directly characterizes physical deflection of the fixtured object and is therefore useful in practical monitoring of manufacturing tolerances. 
We illustrated the planning of minimum deflection fixtures on polygonal objects. The qualitative properties of such fixtures were characterized as follows. In order to reduce object deflection, the fixels should be placed on evenly oriented edges, should spread apart with respect to the fixture's center of compliance, and should minimize the distance from the object's centroid to the fixture's center of compliance. Similar observations have been previously made for rigid [34] as well as compliant grasps [29]. Therefore, it appears that these geometric features are generally possessed by "good" fixture arrangements. We also considered the computation of minimum deflection fixtures for polygonal objects. In the case of three-contact fixtures, we presented a graphical technique for determining the optimal contact arrangement. In the case of four-contact fixtures, an indefinite quadratic programming (IQP) algorithm was devised to find the global minimum-deflection fixture arrangement. This algorithm is practically useful, since the IQP problem associated with four-contact fixtures involves only four independent variables.

Finally, we mention issues that need to be addressed in future research. First and foremost, the deflection quality measure need to be verified in experiments. A high-precision fixturing system is being developed for this purpose, and initial results obtained with this system are reported in [3]. Second, we only used the rms velocity and wrench norms in computing minimum deflection fixtures. Alternative norms such as the max norm should also be explored. The max velocity norm is particularly attractive for fixture planning, due to its physical interpretation as the maximal displacement of object points which are critical for precision monitoring. Lastly, the deflection quality measure is valid for both 2-D and 3-D objects. However, we only discussed optimal fixturing techniques for polygonal objects. While these techniques readily extend to 3-D prismatic objects with a polygonal cross section, many industrial parts are truly 3-D. Algorithms for planning minimum deflection fixtures for such objects are important in practice and should be addressed in future research.

\section{APPENDIX A}

\section{DETAILS CONCERNING VELOCITY AND WRENCH NORMS}

This appendix contains two lemmas concerning velocity and wrench norms, as well as a lemma on the computation of the deflection measure $\Delta_{\max }$. The first lemma asserts the frame invariance of the rms velocity norm.

Lemma 1: The rms velocity norm is invariant with respect to choice of world and body frames.

Proof: Let $\mathcal{F}_{W}$ and $\overline{\mathcal{F}}_{W}$ be two choices of world frames, such that the two frames are related by a rigid body displacement $\left(d_{w}, R_{w}\right)$. Similarly, let $\mathcal{F}_{B}$ and $\overline{\mathcal{F}}_{B}$ be two choices of body frames, such that the two frames are related by a displacement $\left(d_{b}, R_{b}\right)$. Let $r, x, R, q$, and $\dot{q}$ denote entities expressed with respect to $\mathcal{F}_{W}$ and $\mathcal{F}_{B}$, and let $\bar{r}, \bar{x}, \bar{R}, \bar{q}$, and $\dot{\bar{q}}$ denote entities expressed with respect to $\overline{\mathcal{F}}_{W}$ and $\overline{\mathcal{F}}_{B}$. By construction, $r=$
$R_{b} \bar{r}+d_{b}$ and $x=R_{w} \bar{x}+d_{w}$. When we substitute for $x$ and $r$ in $x=R r+d$, we get $R=R_{w} \bar{R} R_{b}^{T}$. Moreover, according to (3)

$$
\left(\begin{array}{c}
v \\
\omega
\end{array}\right)=\left(\begin{array}{c}
R_{w}\left(\bar{v}+\left(\bar{R} R_{b}^{T} d_{b}\right) \times \bar{\omega}\right) \\
R_{w} \bar{\omega}
\end{array}\right)
$$

Let $\bar{\nu}$ denote the weighting function in the $\bar{r}$ coordinates. Since $\nu$ and $\bar{\nu}$ represent the same function, we may write $\bar{\nu}(\bar{r})=$ $\nu\left(R_{b} \bar{r}+d_{b}\right)$. Let $\overline{\mathcal{B}}$ denote the region occupied by $\mathcal{B}$ in the $\bar{r}$ coordinates. Substituting for $r, R$, and $(v, \omega)$ in the rms norm gives the following equivalent integration over $\overline{\mathcal{B}}$ :

$$
\begin{aligned}
\|\dot{q}\|_{\mathrm{rms}}^{2}= & \int_{\mathcal{B}} \nu(r)\|\omega \times R r+v\|^{2} d r \\
= & \int_{\overline{\mathcal{B}}} \bar{\nu}(\bar{r}) \|\left(R_{w} \bar{\omega}\right) \times R_{w} \bar{R} R_{b}^{T}\left(R_{b} \bar{r}+d_{b}\right) \\
& \quad+R_{w}\left(\bar{v}+\left(\bar{R} R_{b}^{T} d_{b}\right) \times \bar{\omega}\right) \|^{2} d \bar{r} .
\end{aligned}
$$

Two terms in the integrant cancel each other as follows. Using the identity $R_{w} v_{1} \times R_{w} v_{2}=R_{w}\left(v_{1} \times v_{2}\right)$, we get $\left(R_{w} \bar{\omega}\right) \times$ $R_{w} \bar{R} R_{b}^{T} d_{b}+R_{w}\left(\bar{R} R_{b}^{T} d_{b} \times \bar{\omega}\right)=\overrightarrow{0}$. Hence

$$
\begin{aligned}
\|\dot{q}\|_{\mathrm{rms}}^{2} & =\int_{\overline{\mathcal{B}}} \bar{\nu}(\bar{r})\left\|\left(R_{w} \bar{\omega}\right) \times R_{w} \bar{R} \bar{r}+R_{w} \bar{v}\right\|^{2} d \bar{r} \\
& =\int_{\overline{\mathcal{B}}} \bar{\nu}(\bar{r})\|\bar{\omega} \times \bar{R} \bar{r}+\bar{v}\|^{2} d \bar{r}=\|\dot{\bar{q}}\|_{\mathrm{rms}}^{2}
\end{aligned}
$$

where we used the identities $R_{b}^{T} R_{b}=I, R_{w} v_{1} \times R_{w} v_{2}=$ $R_{w}\left(v_{1} \times v_{2}\right)$, and $R_{w}^{T} R_{w}=I$.

The next lemma gives the conditions under which $\|\dot{q}\|_{\max }$ is a valid velocity norm.

Lemma 2: If $\mathcal{B}$ is a 2-D object $\|\dot{q}\|_{\max }$ is a norm when $\nu$ is strictly positive at two points of $\mathcal{B}$. If $\mathcal{B}$ is a 3 -D object $\|\dot{q}\|_{\max }$ is a norm when $\nu$ is strictly positive at three noncollinear points of $\mathcal{B}$.

Proof: We must verify that $\|\dot{q}\|_{\max }$ is positive definite, linearly homogeneous, and satisfies the triangle inequality. The function defined in (5) is clearly linearly homogeneous in $\dot{q}$. It is also clearly non-negative. Focusing on the 3 -D case, we now verify that $\|\dot{q}\|_{\max }$ vanishes only at $\dot{q}=0$. The condition $\omega \times R r+v=\overrightarrow{0}$ implies that the object point $r$ moves with pure instantaneous rolling. In general, a 3-D object can simultaneously roll only at points which lie along a common spatial line. Since $\nu$ is strictly positive on three noncollinear points, $\mathcal{B}$ must have at least one point $r$ such that $\nu(r)\|\omega \times R r+v\|>0$. Hence, $\|\dot{q}\|_{\max }$ is positive definite. The third property is triangular inequality, which is equivalent to the condition $\left\|\dot{q}_{1}+\dot{q}_{2}\right\|_{\max }^{2} \leq$ $\left(\left\|\dot{q}_{1}\right\|_{\max }+\left\|\dot{q}_{2}\right\|_{\max }\right)^{2}$. A nonnegative function $a(r)$ always satisfies the identity $\left(\max \left\{\mathrm{a}^{1 / 2}(\mathrm{r})\right\}\right)^{2}=\max \{\mathrm{a}(\mathrm{r})\}$. Applying this fact to $\left\|\dot{q}_{1}+\dot{q}_{2}\right\|_{\text {max }}^{2}$ gives

$$
\begin{aligned}
\left\|\dot{q}_{1}+\dot{q}_{2}\right\|_{\max }^{2}=\max _{\mathrm{r} \in \mathcal{B}}\left\{\dot{\mathrm{q}}_{1}^{\mathrm{T}} \mathcal{R N} \mathcal{R}^{\mathrm{T}} \dot{\mathrm{q}}_{1}+2 \dot{\mathrm{q}}_{1}^{\mathrm{T}} \mathcal{R} \mathrm{RN}^{\mathrm{T}} \dot{\mathrm{q}}_{2}\right. \\
\left.+\dot{q}_{2}^{\mathrm{T}} \mathcal{R} N \mathcal{R}^{T} \dot{q}_{2}\right\} .
\end{aligned}
$$

Given three functions $a(r), b(r), c(r), \quad$ it is always true that $\max \{\mathrm{a}(\mathrm{r})+\mathrm{b}(\mathrm{r})+\mathrm{c}(\mathrm{r})\} \leqslant$ 
$\max \{|\mathrm{a}(\mathrm{r})|\}+\max \{|\mathrm{b}(\mathrm{r})|\}+\max \{|\mathrm{c}(\mathrm{r})|\} . \quad$ Applying this identity to (12) gives

$$
\begin{aligned}
\left\|\dot{q}_{1}+\dot{q}_{2}\right\|_{\max }^{2} \leqslant & \max _{\mathrm{r} \in \mathcal{B}}\left\{\dot{\mathrm{q}}_{1}^{\mathrm{T}} \mathcal{R} \mathrm{N} \mathcal{R}^{\mathrm{T}} \dot{\mathrm{q}}_{1}\right\}+2 \max _{\mathrm{r} \in \mathcal{B}} \\
& \left\{\left|\dot{q}_{1}^{T} \mathcal{R} N \mathcal{R}^{T} \dot{q}_{2}\right|\right\}+\max _{\mathrm{r} \in \mathcal{B}}\left\{\dot{\mathrm{q}}_{2}^{\mathrm{T}} \mathcal{R} \mathrm{N} \mathcal{R}^{\mathrm{T}} \dot{\mathrm{q}}_{2}\right\}
\end{aligned}
$$

where we used the fact that $\mathcal{R N R}^{T}$ is positive semi-definite. A closer look at the term $\dot{q}_{1}^{T} \mathcal{R} N \mathcal{R}^{T} \dot{q}_{2}$ reveals that $\mid \dot{q}_{1}^{T} \mathcal{R} N \mathcal{R}^{T} \dot{q}_{2} \leqslant\left(\dot{q}_{1}^{T} \mathcal{R} N \mathcal{R}^{T} \dot{q}_{1}\right)^{1 / 2}\left(\dot{q}_{2}^{T} \mathcal{R} N \mathcal{R}^{T} \dot{q}_{2}\right)^{1 / 2}$. Given non-negative functions $a(r)$ and $b(r)$, it is always true that $\max \{\mathrm{a}(\mathrm{r}) \mathrm{b}(\mathrm{r})\} \leqslant \max \{\mathrm{a}(\mathrm{r})\} \max \{\mathrm{b}(\mathrm{r})\}$. Using this fact and the identity $\left(\max \left\{\mathrm{a}^{1 / 2}(\mathrm{r})\right\}\right)^{2}=\max \{\mathrm{a}(\mathrm{r})\}$, one gets

$$
\begin{aligned}
\left\|\dot{q}_{1}+\dot{q}_{2}\right\|_{\text {max }}^{2} \leqslant & \max _{\mathrm{r} \in \mathcal{B}}\left\{\left(\dot{\mathrm{q}}_{1}^{\mathrm{T}} \mathcal{R N} \mathcal{R}^{\mathrm{T}} \dot{\mathrm{q}}_{1}\right)^{\frac{1}{2}}\right\}^{2} \\
& +2 \max _{\mathrm{r} \in \mathcal{B}}\left\{\left(\dot{\mathrm{q}}_{1}^{\mathrm{T}} \mathcal{R N} \mathcal{R}^{\mathrm{T}} \dot{\mathrm{q}}_{1}\right)^{\frac{1}{2}}\right\} \\
& \times \max _{\mathrm{r} \in \mathcal{B}}\left\{\left(\dot{\mathrm{q}}_{2}^{\mathrm{T}} \mathcal{R N} \mathcal{R}^{\mathrm{T}} \dot{\mathrm{q}}_{2}\right)^{\frac{1}{2}}\right\} \\
& +\max _{\mathrm{r} \in \mathcal{B}}\left\{\left(\dot{\mathrm{q}}_{2}^{\mathrm{T}} \mathcal{R N} \mathcal{R}^{\mathrm{T}} \dot{\mathrm{q}}_{2}\right)^{\frac{1}{2}}\right\}^{2} \\
= & \left\|\dot{q}_{1}\right\|_{\max }^{2}+2\left\|\dot{q}_{1}\right\|_{\max }\left\|\dot{q}_{2}\right\|_{\max }+\left\|\dot{q}_{2}\right\|_{\text {max }}^{2} .
\end{aligned}
$$

Taking the square root of both sides gives the desired triangle inequality.

The final lemma provides a concrete formula for computing the deflection measure $\Delta_{\max }$.

Lemma 5: Let $\mathcal{B}$ have distinguished points $r_{1}, \ldots, r_{m}$ with discrete weights $\nu_{1}, \ldots, \nu_{m}$. The deflection quality measure for the max velocity norm and rms wrench norm is given by

$$
\Delta_{\max }=\left(\max _{1 \leqslant \mathrm{i} \leqslant \mathrm{m}}\left\{\lambda_{\max }\left(\mathrm{M}^{\frac{1}{2}} \mathrm{C}^{\mathrm{T}} \mathcal{R N}_{\mathrm{i}} \mathcal{R}^{\mathrm{T}} \mathrm{CM}^{\frac{1}{2}}\right)\right\}\right)^{\frac{1}{2}}
$$

where $C=K^{-1}, \mathcal{R}=\operatorname{diag}(R, R), M=\sum_{i=1}^{m} \nu_{i}$ $\left(\begin{array}{cc}I & \widehat{r}_{i}^{T} \\ \widehat{r}_{i} & -\widehat{r}^{2}\end{array}\right)$, and $N_{i}=\nu_{i}^{2}\left(\begin{array}{cc}I & \widehat{r}_{i}^{T} \\ \widehat{r}_{i} & -\widehat{r}^{2}\end{array}\right)$ for $i=1, \ldots, m$. Moreover, the reciprocal deflection quality measure is given by $Q_{\max }=1 / \Delta_{\max }$.

Proof: Using (6), the discretized version of the $\max$ velocity norm is given by $\|\dot{q}\|_{\max }=$ $\max _{1 \leqslant \mathrm{i} \leqslant \mathrm{m}}\left\{\left(\dot{\mathrm{q}}^{\mathrm{T}} \mathcal{R} \mathrm{N}_{\mathrm{i}} \mathcal{R}^{\mathrm{T}} \dot{\mathrm{q}}\right)^{1 / 2}\right\}$. Using the identity $\left(\max \left\{\mathrm{a}^{1 / 2}(\mathrm{r})\right\}\right)^{2}=\max \{\mathrm{a}(\mathrm{r})\}$ and substituting $\dot{q}=C \boldsymbol{w}$ in $\|\dot{q}\|_{\max }$, gives

$$
\begin{aligned}
\Delta_{\max }^{2} & =\max _{\||\boldsymbol{w}|\|_{\mathrm{rms}} \leqslant 1}\left\{\|\mathrm{C} \boldsymbol{w}\|_{\max }^{2}\right\} \\
& =\max _{\boldsymbol{w}^{\mathrm{T}} \mathrm{M}^{-1} \boldsymbol{w} \leqslant 1}\left\{\max _{1 \leqslant \mathrm{i} \leqslant \mathrm{m}}\left\{\boldsymbol{w}^{\mathrm{T}} \mathrm{C}^{\mathrm{T}} \mathcal{R} \mathrm{N}_{\mathrm{i}} \mathcal{R}^{\mathrm{T}} \mathrm{C} \boldsymbol{w}\right\}\right\} .
\end{aligned}
$$

Exchanging $\max _{\|\boldsymbol{w}\|_{\mathrm{rms}} \leqslant 1}\{\cdot\}$ with $\max _{1 \leqslant \mathrm{i} \leqslant \mathrm{m}}\{\cdot\}$ and substituting $x=M^{-1 / 2} w$ gives

$$
\begin{aligned}
\Delta_{\text {max }}^{2} & =\max _{1 \leqslant \mathrm{i} \leqslant \mathrm{m}} \max _{\|\mathrm{x}\| \leqslant 1}\left\{\mathrm{x}^{\mathrm{T}} \mathrm{M}^{\frac{1}{2}} \mathrm{C}^{\mathrm{T}} \mathcal{R} \mathrm{N}_{\mathrm{i}} \mathcal{R}^{\mathrm{T}} \mathrm{CM}^{\frac{1}{2}} \mathrm{x}\right\} \\
& =\max _{1 \leqslant \mathrm{i} \leqslant \mathrm{m}}\left\{\lambda_{\max }\left(\mathrm{M}^{\frac{1}{2}} \mathrm{C}^{\mathrm{T}} \mathcal{R} \mathrm{N}_{\mathrm{i}} \mathcal{R}^{\mathrm{T}} \mathrm{CM}^{\frac{1}{2}}\right)\right\} .
\end{aligned}
$$

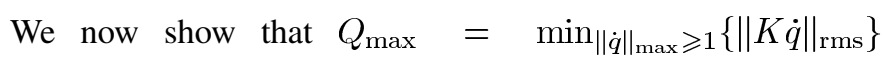
is the reciprocal of $\Delta_{\max }$. By definition, $\|\mid K \dot{q}\|_{\mathrm{rms}}=$ $\left(\dot{q}^{T} K^{T} M^{-1} K \dot{q}\right)^{1 / 2}$. Substituting $x=M^{-1 / 2} K \dot{q}$ and using the identity $\left(\max \left\{\mathrm{a}^{1 / 2}(\mathrm{r})\right\}\right)^{2}=\max \{\mathrm{a}(\mathrm{r})\}$ gives $Q_{\max }^{2}=\min _{\left\|C M^{1 / 2} x\right\|_{\max } \geqslant 1}\left\{\|x\|^{2}\right\}$, where $C=K^{-1}$ and $\|\cdot\|$ is the usual Euclidean norm. The extrema of $\|x\|^{2}$ are located on the boundary of the constraint set, given by $\mathcal{S}=\left\{x:\left\|C M^{1 / 2} x\right\|_{\max }=1\right\}$. Furthermore, it can be verified that $\left\|C M^{1 / 2} x\right\|_{\max }$ is a convex function of $x$. Hence, $\mathcal{S}$ is convex. This implies that the extrema of $\|x\|^{2}$ occur on the individual patches that comprise the set $\mathcal{S}$. The $i$ th patch is a subset of the quadratic hyper-surface $x^{T} P_{i} x=1$, where $P_{i}=M^{1 / 2} C^{T} \mathcal{R} N_{i} \mathcal{R}^{T} C M^{1 / 2}$ for $i=1, \ldots, m$. An extremum of $\|x\|^{2}$ on the $i$ th patch satisfies the condition

$$
P_{i} x=\lambda x \text { for some } \lambda \in \mathbb{R} .
$$

The extrema of $\|x\|^{2}$ on the $i$ th patch are therefore eigenvectors of $P_{i}$ with an eigenvalue $\lambda$. Multiplying both sides of (13) by $x$, we obtain that $x^{T} P_{i} x=\lambda\|x\|^{2}=1$. It follows that $\|x\|^{2}=1 / \lambda$ at the extrema of $\|x\|^{2}$ on $\mathcal{S}$. Let $i_{0}$ be the index of the matrix $P_{i}$ with the largest maximal eigenvalue, $\lambda_{\max }\left(P_{i_{0}}\right)=\max _{1 \leqslant \mathrm{i} \leqslant \mathrm{m}}\left\{\lambda_{\max }\left(\mathrm{P}_{\mathrm{i}}\right)\right\}$. Let $v$ be the corresponding eigenvector, such that $v^{T} P_{i_{0}} v=1$. Since $v^{T} P_{i_{0}} v \geqslant v^{T} P_{i} v$ for $i=1, \ldots, m$, the eigenvector $v$ must lie on the $i_{0}^{\text {th }}$ patch of $\mathcal{S}$. Since $\lambda_{\max }\left(P_{i_{0}}\right)$ is the largest possible eigenvalue and $\|x\|^{2}=1 / \lambda$, the global minimum of $\|x\|^{2}$ on the set $\mathcal{S}$ is $1 / \lambda_{\max }\left(P_{i_{0}}\right)$. Thus, $Q_{\max }^{2}=1 / \lambda_{\max }\left(P_{i_{0}}\right)=1 / \Delta_{\max }^{2}$.

\section{APPENDIX B}

\section{Details Concerning Optimal Fixtures of Polygons}

This appendix contains proofs of two key propositions concerned with optimal fixturing of polygons by three and four fixels. Proposition 8 provides an approximate expression for $Q_{\text {rms }}$ in three-contact fixtures. In order to prove the proposition, we need to compare orders of magnitude of the stiffness parameters $\sigma_{1}, \sigma_{2}$, and $\widetilde{\mu}=\mu / \rho^{2}$, which have the same dimension. The parameters $\sigma_{1}$ and $\sigma_{2}$ are the eigenvalues of the matrix $K_{11}=\sum_{i=1}^{3} k_{i} n_{i} n_{i}^{T}$. Excluding degenerate edge triplets with almost parallel edges, it can be verified that these eigenvalues have the same order of magnitude as the contact stiffnesses $k_{i}$. Thus, $\sigma_{1}, \sigma_{2} \cong k_{i}$ for $i=1,2,3$. The radius of $\mathcal{B}$ with respect to $\nu$ satisfies $\rho \cong a$ and $\rho \cong r$. Using Lemma 7, it follows that $\mu / \rho^{2}=f_{T}(2 a \gamma+r) / \rho^{2} \cong f_{T} / a$. Now we invoke the basic fact that deformations at a quasirigid contact are much smaller than the object's characteristic dimension. ${ }^{7}$ The deformation at the $i^{\text {th }}$ contact is on the order of magnitude of $f_{T} / k_{i}$. Identifying $\mathcal{B}$ 's characteristic dimension with $a$, we have that $f_{T} / k_{i} \ll a$, or equivalently $f_{T} / a \ll k_{i}$. Since $\sigma_{1}, \sigma_{2} \cong k_{i}$, we obtain that $\mu / \rho^{2} \ll \min \left\{\sigma_{1}, \sigma_{2}\right\}$, or equivalently $\widetilde{\mu} \ll \min \left\{\sigma_{1}, \sigma_{2}\right\}$. We also need the following lemma on eigenvalue perturbation.

Lemma 13 ([11]): Let a real symmetric matrix $A$ be perturbed to $A+B$, where $B$ is real symmetric with $\|B\| \ll\|A\|$. Let $\lambda$ be a distinct eigenvalue of $A$ with an associated unit-magnitude eigenvector $x$. Then, $\lambda+x^{T} B x$ is an eigenvalue of the perturbed matrix $A+B$.

The inequality $\widetilde{\mu} \ll \min \left\{\sigma_{1}, \sigma_{2}\right\}$ and Lemma 13 allow us to prove Proposition 8.

\footnotetext{
${ }^{7}$ This fact holds true under any quasirigid contact model, including highly nonlinear models where the coefficients $k_{i}$ depend on $f_{T}$.
} 
Proof of Proposition 8: We can write the scaled stiffness matrix as $\widetilde{K}=\widetilde{K}_{1}+\widetilde{K}_{2}$, where

$$
\begin{aligned}
& \widetilde{K}_{1}=\left(\begin{array}{ccc}
\sigma_{1} & 0 & -\sigma_{1} \tilde{\eta} \\
0 & \sigma_{2} & \sigma_{2} \tilde{\xi} \\
-\sigma_{1} \widetilde{\eta} & \sigma_{2} \widetilde{\xi} & \sigma_{2} \widetilde{\xi}^{2}+\sigma_{1} \widetilde{\eta}^{2}
\end{array}\right) \text { and } \\
& \widetilde{K}_{2}=\left(\begin{array}{ccc}
0 & & \\
& 0 & \\
& & \widetilde{\mu}
\end{array}\right) .
\end{aligned}
$$

Since $\widetilde{\mu} \ll \min \left\{\sigma_{1}, \sigma_{2}\right\}, \widetilde{K}_{2}$ can be viewed as a small perturbation to $\widetilde{K}_{1}$. Now, $\widetilde{K}_{1}$ is positive semidefinite with a distinct eigenvalue $\lambda=0$ and a corresponding eigenvector $(\widetilde{\eta},-\widetilde{\xi}, 1)$. Applying Lemma 13 using this eigenvector, $x^{T} B x=\widetilde{\mu} /\left(\widetilde{\xi}^{2}+\right.$ $\left.\widetilde{\eta}^{2}+1\right)=\mu /\left(\rho^{2}+z^{2}\right)$ is approximately an eigenvalue of $\widetilde{K}$. (The denominator $\widetilde{\xi}^{2}+\widetilde{\eta}^{2}+1$ is due to a normalization of the eigenvector $(\widetilde{\eta},-\widetilde{\xi}, 1)$, and $z^{2}=\xi^{2}+\mu^{2}$.) To show that this is the smallest eigenvalue of $\widetilde{K}$, note that regardless of the values of $\widetilde{\xi}$ and $\widetilde{\eta}$, the nonzero eigenvalues of $\widetilde{K}_{1}$ are no less than $\min \left\{\sigma_{1}, \sigma_{2}\right\}$. Also, it can be shown that $\sigma_{1}$ and $\sigma_{2}$ are perturbed to give the remaining eigenvalues of $\widetilde{K}$, and the perturbations are on the order of the matrix norm $\left\|\widetilde{K}_{2}\right\|=\widetilde{\mu}$. Since $\widetilde{\mu} \ll \min \left\{\sigma_{1}, \sigma_{2}\right\}$, it follows that $\mu /\left(\rho^{2}+z^{2}\right)$ is the smallest eigenvalue of $\widetilde{K}$.

Next, consider Lemma 11, which provides a lower bound for $Q_{\mathrm{rms}}$ in four-contact fixtures.

Lemma 11: Let a polygonal object $\mathcal{B}$ be held by four fixels. Let $\rho$ be $\mathcal{B}$ 's radius associated with the weighting function $\nu$. As the contacts vary along a particular edge combination, $Q_{\mathrm{rms}}$ satisfies the lower bound

$$
\begin{aligned}
Q_{\mathrm{rms}}(\boldsymbol{s}) \geqslant \min \left\{\sigma_{1},\right. & \sigma_{2}, \frac{1}{2}\left(\sigma \widetilde{z}^{2}(\boldsymbol{s})+\widetilde{\mu}(\boldsymbol{s})+\sigma\right. \\
& \left.\left.-\sqrt{\left(\sigma \widetilde{z}^{2}(\boldsymbol{s})+\widetilde{\mu}(\boldsymbol{s})+\sigma\right)^{2}-4 \sigma \widetilde{\mu}(\boldsymbol{s})}\right)\right\}
\end{aligned}
$$

where $\sigma=\min \left\{\sigma_{1}, \sigma_{2}\right\}, \widetilde{\mu}=\mu(\boldsymbol{s}) / \rho^{2}$, and $\widetilde{z}=z(\boldsymbol{s}) / \rho$ where $z(\boldsymbol{s})$ is the distance between the fixture's center of compliance and $\mathcal{B}$ 's centroid. Moreover, the lower bound is monotonically increasing in $\mu(\boldsymbol{s})$ and monotonically decreasing in $z(\boldsymbol{s})$.

Proof: By definition, $Q_{\mathrm{rms}}=\lambda_{\min }(\widetilde{K})$. Let us assume that $\sigma_{1} \leqslant \sigma_{2}$. Then, the scaled stiffness matrix can be written as the following sum of two matrices:

$$
\begin{aligned}
\widetilde{K} & =\left(\begin{array}{ccc}
\sigma_{1} & 0 & -\sigma_{1} \widetilde{\eta} \\
0 & \sigma_{2} & \sigma_{2} \widetilde{\xi} \\
-\sigma_{1} \widetilde{\eta} & \sigma_{2} \widetilde{\xi} & \widetilde{\mu}+\sigma_{2} \widetilde{\xi}^{2}+\sigma_{1} \widetilde{\eta}^{2}
\end{array}\right) \\
& =\left(\begin{array}{ccc}
\sigma_{1} & 0 & -\sigma_{1} \widetilde{\eta} \\
0 & \sigma_{1} & \sigma_{1} \widetilde{\xi} \\
-\sigma_{1} \widetilde{\eta} & \sigma_{1} \widetilde{\xi} & \widetilde{\mu}+\sigma_{1} \widetilde{z}^{2}
\end{array}\right)+\left(\sigma_{2}-\sigma_{1}\right)\left(\begin{array}{ccc}
0 & 0 & 0 \\
0 & 1 & \widetilde{\xi} \\
0 & \widetilde{\xi} & \widetilde{\xi}^{2}
\end{array}\right)
\end{aligned}
$$

where $\widetilde{z}^{2}=\widetilde{\xi}^{2}+\widetilde{\eta}^{2}$. Since $\sigma_{1} \leqslant \sigma_{2}$, both matrices are positive semidefinite. Hence, $\lambda_{\min }(\widetilde{K})$ satisfies the lower bound

$$
Q_{\mathrm{rms}}=\lambda_{\min }(\widetilde{K}) \geqslant \lambda_{\min }\left(\begin{array}{ccc}
\sigma & 0 & -\sigma \widetilde{\eta} \\
0 & \sigma & \sigma \widetilde{\xi} \\
-\sigma \widetilde{\eta} & \sigma \widetilde{\xi} & \widetilde{\mu}+\sigma \widetilde{z}^{2}
\end{array}\right)
$$

where we replaced $\sigma_{1}$ with $\sigma=\min \left\{\sigma_{1}, \sigma_{2}\right\}$. The eigenvalues of the latter matrix are given by $\lambda_{1}=\sigma$ and $\lambda_{2,3}=(1 / 2)\left(\sigma \widetilde{z}^{2}+\right.$ $\widetilde{\mu}+\sigma \pm \sqrt{\left(\sigma \widetilde{z}^{2}+\widetilde{\mu}+\sigma\right)^{2}-4 \sigma \widetilde{\mu}}$, and the lower bound on $Q_{\mathrm{rms}}$ is specified in terms of these eigenvalues.

Next, we verify that the lower bound is monotonically decreasing in $\widetilde{z}$, which would imply the same monotonicity in $z$. Let $f(\widetilde{z}, \widetilde{\mu})=\sigma \widetilde{z}^{2}+\widetilde{\mu}+\sigma-\sqrt{\left(\sigma \widetilde{z}^{2}+\widetilde{\mu}+\sigma\right)^{2}-4 \sigma \widetilde{\mu}}$. Since $Q_{\mathrm{rms}}=\min \{\sigma, f(\widetilde{z}, \widetilde{\mu})\}$, we only need to verify monotonically for the case where $f(\widetilde{z}, \widetilde{\mu}) \leqslant \sigma$. Thus

$$
\frac{\partial}{\partial \widetilde{z}} f(\widetilde{z}, \widetilde{\mu})=2 \sigma \widetilde{z}\left(1-\frac{\sigma \widetilde{z}^{2}+\widetilde{\mu}+\sigma}{\sqrt{\left(\sigma \widetilde{z}^{2}+\widetilde{\mu}+\sigma\right)^{2}-4 \sigma \widetilde{\mu}}}\right)<0
$$

since $\sigma \widetilde{z}>0$ (except at $\widetilde{z}=0$ ), and the square-root satisfies $\sqrt{\left(\sigma \widetilde{z}^{2}+\widetilde{\mu}+\sigma\right)^{2}-4 \sigma \widetilde{\mu}}<\left|\sigma \widetilde{z}^{2}+\widetilde{\mu}+\sigma\right|$. Finally we verify that the lower bound increases monotonically in $\widetilde{\mu}$, which would imply the same monotonicity in $\mu$.

$$
\begin{aligned}
\frac{\partial}{\partial \widetilde{\mu}} f(\widetilde{z}, \widetilde{\mu}) & =1-\frac{\left(\sigma \widetilde{z}^{2}+\widetilde{\mu}+\sigma\right)-4 \sigma}{\sqrt{\left(\sigma \widetilde{z}^{2}+\widetilde{\mu}+\sigma\right)^{2}-4 \sigma \widetilde{\mu}}} \\
& =1-\frac{\left(\sigma \widetilde{z}^{2}+\widetilde{\mu}\right)-3 \sigma}{\sqrt{\left(\sigma \widetilde{z}^{2}+\widetilde{\mu}+\sigma\right)^{2}-4 \sigma \widetilde{\mu}}} .
\end{aligned}
$$

By assumption, $f(\widetilde{z}, \widetilde{\mu}) \leqslant \sigma$. Substituting for $f$ in this inequality $\sigma \widetilde{z}^{2}+\widetilde{\mu}+\sigma-\sqrt{\left(\sigma \widetilde{z}^{2}+\widetilde{\mu}+\sigma\right)^{2}-4 \sigma \widetilde{\mu}} \leqslant \sigma$. Hence $\left(\sigma \widetilde{z}^{2}+\widetilde{\mu}\right) / \sqrt{\left(\sigma \widetilde{z}^{2}+\widetilde{\mu}+\sigma\right)^{2}-4 \sigma \widetilde{\mu}} \leqslant 1$. It follows that the partial derivative of $f$ with respect to $\widetilde{\mu}$ satisfies the inequality

$$
\frac{\partial}{\partial \widetilde{\mu}} f(\widetilde{z}, \widetilde{\mu}) \geqslant \frac{3 \sigma}{\sqrt{\left(\sigma \widetilde{z}^{2}+\widetilde{\mu}+\sigma\right)^{2}-4 \sigma \widetilde{\mu}}}>0
$$

since $\sigma>0$.

Finally, consider Proposition 12, which gives a procedure for computing the maximal value of $Q_{\mathrm{rms}}$ based on an auxiliary function $\psi$. We first give a preliminary lemma. Without loss of generality, let $\sigma_{1} \leqslant \sigma_{2}$, and recall the notation $c=\sigma_{1} / \sigma_{2}$. For a parameter $t \in(0,1)$, we define

$$
\begin{aligned}
\zeta(t, \boldsymbol{s})= & \widetilde{\mu}(\boldsymbol{s})+\left(1-\frac{1}{1-c(1-t)}\right) \sigma_{2} \widetilde{\xi}^{2}(\boldsymbol{s}) \\
& +\left(1-\frac{1}{t}\right) \sigma_{1} \widetilde{\eta}^{2}(\boldsymbol{s}), \\
\zeta_{c}(t, \boldsymbol{s})= & \frac{1}{1-c(1-t)} \sigma_{2} \widetilde{\xi}^{2}(\boldsymbol{s})+\frac{1}{t} \sigma_{1} \widetilde{\eta}^{2}(\boldsymbol{s}) .
\end{aligned}
$$

Since $0<c \leqslant 1$, we have that $1-c(1-t)>0$, and the functions $\zeta$ and $\zeta_{c}$ are therefore well defined. The following decomposition of $\widetilde{K}$ can then be verified $\widetilde{K}(\boldsymbol{s})=\widetilde{K}_{1}(t, \boldsymbol{s})+$ $\widetilde{K}_{2}(t, \boldsymbol{s})$, where

$$
\begin{aligned}
\widetilde{K}_{1}(t, \boldsymbol{s}) & =\operatorname{diag}\left((1-t) \sigma_{1},(1-t) \sigma_{1}, \zeta(t, \boldsymbol{s})\right) \\
\widetilde{K}_{2}(t, \boldsymbol{s}) & =\left(\begin{array}{ccc}
t \sigma_{1} & 0 & -\sigma_{1} \widetilde{\eta}(\boldsymbol{s}) \\
0 & (1-c(1-t)) \sigma_{2} & \sigma_{2} \widetilde{\xi}(\boldsymbol{s}) \\
-\sigma_{1} \widetilde{\eta}(\boldsymbol{s}) & \sigma_{2} \widetilde{\xi}(\boldsymbol{s}) & \zeta_{c}(t, \boldsymbol{s})
\end{array}\right) .
\end{aligned}
$$

The symmetry of $\widetilde{K}_{1}$ and $\widetilde{K}_{2}$ implies that $\lambda_{\min }\left(\widetilde{K}_{1}\right)+$ $\lambda_{\min }\left(\widetilde{K}_{2}\right) \leqslant \lambda_{\min }\left(\widetilde{K}_{1}+\widetilde{K}_{2}\right) \leqslant \lambda_{\max }\left(\widetilde{K}_{1}\right)+\lambda_{\min }\left(\widetilde{K}_{2}\right)$. 
Furthermore, it can be shown that $\widetilde{K}_{2}$ is positive semidefinite, and $\lambda_{\min }\left(\widetilde{K}_{2}\right)=0$. Thus

$$
\begin{aligned}
\min & \left\{(1-t) \sigma_{1}, \zeta(t, \boldsymbol{s})\right\} \\
& \leqslant \lambda_{\min }(\widetilde{K}(\boldsymbol{s})) \\
& \leqslant \max \left\{(1-t) \sigma_{1}, \zeta(t, \boldsymbol{s})\right\}, \quad \text { for all } 0<t<1
\end{aligned}
$$

These bounds allow the following characterization of $\lambda_{\min }(\tilde{K})$.

Lemma 14: For a fixed $\boldsymbol{s} \in \mathcal{T}$, the function $\zeta(t, \boldsymbol{s})$ is monotonically increasing in $t$, and either of the following two cases must be true.

1) There is a unique $t^{*} \in(0,1)$, such that $\lambda_{\min }(\widetilde{K}(\boldsymbol{s}))=$ $\left(1-t^{*}\right) \sigma_{1}=\zeta\left(t^{*}, \boldsymbol{s}\right)$.

2) $\lambda_{\min }(\widetilde{K}(\boldsymbol{s}))$ achieves the upper bound $\sigma_{1}$ (i.e., $\left.\lambda_{\min }(\widetilde{K}(\boldsymbol{s}))=\sigma_{1}\right)$.

Proof: Since $(\partial \zeta / \partial t)(t, s)=\left(\sigma_{1} \widetilde{\xi}^{2} /(1-c(1-t))^{2}\right)+$ $\left(\sigma_{1} \widetilde{\eta}^{2} / t^{2}\right)>0$, the function $\zeta(t, \boldsymbol{s})$ is monotonically increasing in $t$. Consider the function (with dependence on $\boldsymbol{s}$ suppressed)

$$
\begin{aligned}
\phi(t) & =\zeta(t)-(1-t) \sigma_{1} \\
& =\widetilde{\mu}+\left(1-\frac{1}{1-c(1-t)}\right) \sigma_{2} \widetilde{\xi}^{2}+\left(1-\frac{1}{t}\right) \sigma_{1} \widetilde{\eta}^{2}-(1-t) \sigma_{1}
\end{aligned}
$$

which is also monotonically increasing in $t$. If $\widetilde{\eta} \neq 0$, then $\phi\left(0^{+}\right)=-\infty$ and $\phi(1)=\widetilde{\mu}>0$. Hence, $\phi$ has a unique zero in the interval $(0,1)$. This fact, along with the bounds in (14), leads to Case (1). It remains to consider the case $\widetilde{\eta} \equiv 0$. In this case, $\widetilde{K}=\left(\begin{array}{cc}\sigma_{1} & 0 \\ 0 & D\end{array}\right)$, where $D=\left(\begin{array}{cc}\sigma_{2} & \sigma_{2} \widetilde{\xi} \\ \sigma_{2} \widetilde{\xi} & \widetilde{\mu}+\sigma_{2} \widetilde{\xi}^{2}\end{array}\right)$. The characteristic equation of the positive definite matrix $D$ is given by $\bar{\phi}(z)=z^{2}-\left(\widetilde{\mu}+\sigma_{2}+\sigma_{2} \widetilde{\xi}^{2}\right) z+\sigma_{2} \widetilde{\mu}=0$, which transforms to the equation $\phi(t)=0$ by change of variable $z=(1-t) \sigma_{1}$. Thus, there is a one-to-one correspondence between the zeros of $\phi(t)$ and the eigenvalues of $D$. Now, $\lambda_{\max }(D)=\sigma_{2}\left(1+\widetilde{\xi}^{2}\right) \geqslant \sigma_{2} \geqslant \sigma_{1}$. Hence, if $\lambda_{\min }(D)<\sigma_{1}$, it is the only eigenvalue of $D$ in the interval $\left(0, \sigma_{1}\right)$. This implies that $\bar{\phi}$ has a unique zero in $\left(0, \sigma_{1}\right)$ and, consequently, $\phi$ has a unique zero in $(0,1)$. This is again Case (1). Otherwise, we have $\lambda_{\min }(D) \geqslant \sigma_{1}$ and $\lambda_{\min }(\widetilde{K}(\boldsymbol{s}))=\sigma_{1}$, which is Case (2).

It follows from the lemma that maximizing $\lambda_{\min }(\widetilde{K}(\boldsymbol{s}))$ over $\mathcal{T}$ is equivalent to minimizing the scalar $t$ over the constraint $\zeta(t, \boldsymbol{s})=(1-t) \sigma_{1}$ such that $t \in(0,1)$ and $\boldsymbol{s} \in \mathcal{T}$. Moreover, if no such $t$ exists, the maximal value of $\lambda_{\min }(\widetilde{K})$ is precisely $\sigma_{1}$. Defining $\psi(t)=\max _{\boldsymbol{s} \in \mathcal{T}}\{\phi(\mathrm{t}, \boldsymbol{s})\}$, where $\phi(t, \boldsymbol{s})=\zeta(t, \boldsymbol{s})-$ $(1-t) \sigma_{1}$, we are now in a position to prove Proposition 12 .

Proof of Proposition 12: According to Lemma 14, the function $\zeta(t, \boldsymbol{s})$ and, consequently, $\phi(t, \boldsymbol{s})$ is increasing in $t$ in the interval $(0,1)$. Hence, $\psi(t)=\max _{\boldsymbol{s} \in \mathcal{T}}\{\phi(\mathrm{t}, \boldsymbol{s})\}$ is also increasing in $t$ in the interval $(0,1)$. This implies that $\psi$ has at most one root in the interval $(0,1)$. We now prove the second part of the proposition, starting with Case (1). Suppose that $\psi$ has a unique zero $t^{*} \in(0,1)$. Then, $\zeta\left(t^{*}, \boldsymbol{s}^{*}\right)=\left(1-t^{*}\right) \sigma_{1}$ for some $s^{*} \in \mathcal{T}$. Using Lemma 14, we have

$$
\begin{aligned}
\lambda_{\min }(\widetilde{K}(\boldsymbol{s})) & \leqslant \max \left\{\left(1-\mathrm{t}^{*}\right) \sigma_{1}, \zeta\left(\mathrm{t}^{*}, \boldsymbol{s}\right)\right\} \\
& \leqslant \max \left\{\left(1-\mathrm{t}^{*}\right) \sigma_{1}, \max _{\boldsymbol{s} \in \mathcal{T}} \zeta\left(\mathrm{t}^{*}, \boldsymbol{s}\right)\right\} \\
& =\left(1-t^{*}\right) \sigma_{1} .
\end{aligned}
$$

But from the same lemma, $\lambda_{\min }\left(\widetilde{K}\left(\boldsymbol{s}^{*}\right)\right)=\left(1-t^{*}\right) \sigma_{1}$. Therefore, $\boldsymbol{s}^{*}$ is the desired optimal fixture. Conversely, let $\boldsymbol{s}^{*}$ be the optimal fixture such that for any $\boldsymbol{s} \in \mathcal{T}$, we have $\lambda_{\min }(\widetilde{K}(\boldsymbol{s})) \leqslant$ $\lambda_{\min }\left(\widetilde{K}\left(\boldsymbol{s}^{*}\right)\right)<\sigma_{1}$. By Lemma 14 , there exists $t^{*}$ and $t$ in $(0,1)$, with $t^{*} \leqslant t$, such that $\lambda_{\min }\left(\widetilde{K}\left(\boldsymbol{s}^{*}\right)\right)=\zeta\left(t^{*}, \boldsymbol{s}^{*}\right)=\left(1-t^{*}\right) \sigma_{1}$, and $\lambda_{\min }(\widetilde{K}(\boldsymbol{s}))=\zeta(t, \boldsymbol{s})=(1-t) \sigma_{1}$. This implies that

$$
\zeta\left(t^{*}, \boldsymbol{s}\right) \leqslant \zeta(t, \boldsymbol{s})=(1-t) \sigma_{1} \leq\left(1-t^{*}\right) \sigma_{1}=\zeta\left(t^{*}, \boldsymbol{s}^{*}\right) .
$$

Therefore, $\max _{\boldsymbol{s}}\left\{\zeta\left(\mathrm{t}^{*}, \boldsymbol{s}\right)\right\}=\zeta\left(\mathrm{t}^{*}, \boldsymbol{s}^{*}\right)=\left(1-\mathrm{t}^{*}\right) \sigma_{1}$. Since $\phi(t, \boldsymbol{s})=\zeta(t, \boldsymbol{s})-(1-t) \sigma_{1}$, we obtain that $\psi\left(t^{*}\right)=\max _{\boldsymbol{s}}\left\{\phi\left(\mathrm{t}^{*}, \boldsymbol{s}\right)\right\}=0$.

Finally consider Case (2) in the proposition. If $\psi$ has no root in $(0,1)$, then suppose that $\max _{\boldsymbol{s}}\left\{\lambda_{\min }(\widetilde{\mathrm{K}}(\boldsymbol{s}))\right\}<\sigma_{1}$. From Case (1), $\psi$ would have a zero in $(0,1)$, a contradiction. Conversely, let $\max _{\boldsymbol{s}}\left\{\lambda_{\min }(\widetilde{\mathrm{K}}(\boldsymbol{s}))\right\}=\sigma_{1}$. If $\psi$ had a zero in $(0,1)$, then Case (1) would imply that $\max _{\boldsymbol{s}}\left\{\lambda_{\min }(\widetilde{\mathrm{K}}(\boldsymbol{s}))\right\}<\sigma_{1}$, again a contradiction. Thus, $\max _{\boldsymbol{s}}\left\{\lambda_{\min }(\widetilde{\mathrm{K}}(\boldsymbol{s}))\right\}=\sigma_{1}$. To prove the second assertion in this case, let $\boldsymbol{s}^{*} \in \mathcal{T}$ be such that $\zeta\left(t, \boldsymbol{s}^{*}\right)=$ $\max _{\boldsymbol{s}}\{\zeta(\mathrm{t}, \boldsymbol{s})\}$ for a given $t \in(0,1)$. Clearly, $\lambda_{\min }\left(\widetilde{K}\left(\boldsymbol{s}^{*}\right)\right) \leqslant$ $\sigma_{1}$. Suppose that $\zeta\left(t, \boldsymbol{s}^{*}\right)<(1-t) \sigma_{1}$. Thus, $\zeta(t, \boldsymbol{s})<(1-t) \sigma_{1}$ for all $s \in \mathcal{T}$. According to the upper bound in (14), this implies that $\lambda_{\min }(\widetilde{K}(\boldsymbol{s})) \leqslant(1-t) \sigma_{1}<\sigma_{1}$ for all $\boldsymbol{s} \in \mathcal{T}$ - a contradiction. Hence, $\zeta\left(t, \boldsymbol{s}^{*}\right) \geqslant(1-t) \sigma_{1}$, and by the lower bound in (14), we obtain $\lambda_{\min }\left(K\left(\boldsymbol{s}^{*}\right)\right) \geqslant(1-t) \sigma_{1}$.

\section{REFERENCES}

[1] A. Bicchi, "On the closure properties of robotic grasping," Int. J. Robot. Res., vol. 14, no. 4, pp. 319-334, Aug. 1995.

[2] — , "Hands for dexterous manipulation and robust grasping: a difficult road toward simplicity," IEEE Trans. Robot. Automat., vol. 16, no. 6, pp. 652-662, Dec. 2000.

[3] J. W. Burdick, Y. Liang, and E. Rimon, "Experiments in fixturing mechanics," in Proc. IEEE Int. Conf. Robotics Automation, 2003, pp. 2579-2585.

[4] M. Buss, L. Faybusovich, and J. Moore, "Dikin-type algorithms for dextrous grasping force optimization," Int. J. Robot. Res., vol. 17, no. 8, pp. 311-334, Aug. 1998.

[5] P. Campbell, Basic Fixture Design. New York: Industrial Press, 1994.

[6] Y.-C. Chou, V. Chandru, and M. M. Barash, "A mathematical approach to automatic configuration of machining fixtures: analysis and synthesis," ASME J. Eng. Ind., vol. 111, pp. 299-306, Nov. 1989.

[7] M. R. Cutkosky and I. Kao, "Computing and controlling the compliance of a robotic hand," IEEE Trans. Robot. Automat., vol. 5, no. 2, pp. 151-165, Apr. 1989.

[8] J. P. Donoghue, W. S. Howard, and V. Kumar, "Stable workpiece fixturing," in Proc. ASME Design Technical Conf., vol. DE 69-2, 1994, pp. $475-482$.

[9] C. Ferrari and J. C. Canny, "Planning optimal grasps," in Proc. Int. Conf. Robotics Automation, Nice, France, May 1992, pp. 2290-2295.

[10] C. A. Floudas and V. Visweswaran, "Quadratic optimization," in Handbook of Global Optimization, R. Horst and P. M. Pardalos, Eds. Norwell, MA: Kluwer, 1995.

[11] J. N. Franklin, Matrix Theory. Upper Saddle River, NJ: Prentice-Hall, 1968.

[12] Y. Funahashi, T. Yamada, M. Tate, and Y. Suzuki, "Grasp stability analysis considering the curvatures at contact points," in Proc. IEEE Int. Conf. Robotics Automation, 1996, pp. 3040-3046.

[13] L. Han, J. C. Trinkle, and Z. X. Li, "Grasp analysis as linear matrix inequality problems," IEEE Trans. Robot. Automat., vol. 16, no. 6, pp. 663-674, Dec. 2000.

[14] F. B. Hazen and P. K. Wright, "Workholding automation: innovations in analysis, design, and planning," Manufact. Rev., vol. 3, no. 4, pp. 224-236, 1990.

[15] H. Hertz, "On the contact of rigid elastic solids and on hardness," in Misc. Papers by H. Hertz. London, U.K.: Jones and Schott, 1896. English. 
[16] M. J. Hockenberger and E. C. De Meter, "Preliminary experimentation and modeling of workpiece displacement during machining," Proc. NAMRI/SME, vol. 21, pp. 351-357, 1993.

[17] _ _ "Effect of machining fixture design parameters on workpiece displacement," Manufact. Rev., vol. 8, no. 1, pp. 22-32, 1995.

[18] E. G. Hoffman, Jig and Fixture Design. Albany, NY: Delmar, 1991.

[19] W. S. Howard and V. Kumar, "Stability of planar grasps," in Int. Conf. Robotics Automation, 1994, pp. 2822-2827.

[20] — , "On the stability of grasped objects," IEEE Trans. Robot. Automat., vol. 12, no. 6, pp. 904-917, Dec. 1996.

[21] Z. Ji and B. Roth, "Direct computation of grasping force for three-finger tip-prehension grasps," ASME J. Mechanisms, Transmissions, Automation in Design, vol. 110, pp. 405-413, 1988.

[22] K. L. Johnson, "One hundred years of hertz contact," in Proc. Inst. Mechanical Engineers, vol. 196, 1982, pp. 363-378.

[23] — Contact Mechanics. Cambridge, U.K.: Cambridge Univ. Press, 1985 .

[24] J. Kerr and B. Roth, "Analysis of multifingered hands," Int. J. Robot. Res., vol. 4, no. 4, pp. 3-17, 1986.

[25] D. G. Kirkpatrick, B. Mishra, and C. K. Yap, "Quantitative Steinitz's theorems with applications to multifingered grasping," in Proc. 20th ACM Symp. Theory Computing, Baltimore, MD, May 1990, pp. 341-351.

[26] Z. Li and S. Sastry, "Task oriented optimal grasping by multifingered robot hands," in Int. Conf. Robotics Automation, Raleigh, NC, Apr. 1987, pp. 389-394.

[27] Q. Lin, "Mechanics and planning of workpiece fixturing and robotic grasping," Ph.D. dissertation, Dept. Mechan. Eng. California Inst. Technol., Pasadena, CA, May 1998.

[28] Q. Lin and J. W. Burdick, "Objective and frame-invariant kinematic metric functions for rigid bodies," Int. J. Robot. Res., vol. 19, no. 6, pp. $612-625,2000$.

[29] Q. Lin, J. W. Burdick, and E. Rimon, "A stiffness based quality measure for compliant grasps and fixtures," IEEE Trans. Robot. Automat., vol. 16, no. 6, pp. 675-688, Dec. 2000.

[30] - "Computation and analysis of natural compliance in grasping and fixturing arrangements," IEEE Trans. Robot. Automat., vol. 20, no. 4, pp. 651-667, Aug. 2004.

[31] X. Markenscoff and C. H. Papadimitriou, "Optimum grip of a polygon," Int. J. Robot. Res., vol. 8, no. 2, pp. 17-29, 1989.

[32] J. M. McCarthy, An Introduction to Theoretical Kinematics. Cambridge, MA: Mass. Inst. Technol., 1990.

[33] R. J. Menassa and W. R. DeVries, "Optimization methods applied to selecting support positions in fixture design," ASME J. Eng. Indust., vol. 113, pp. 412-418, 1991.

[34] B. Mirtich and J. Canny, "Easily computable optimum grasps in 2-D and 3-D," in Int. Conf. Robot. Automat., 1994, pp. 739-747.

[35] B. Mishra, "Workholding," in Proc. IEEE/RSJ Int. Conf. Intelligent Robots Systems, 1991, pp. 53-57.

[36] Y. Nakamura, K. Nagai, and T. Yoshikawa, "Dynamics and stability in coordination of multiple robotic mechanisms," Int. J. Robot. Res., vol. 8, no. 2, pp. 44-59, 1989.

[37] V.-D. Nguyen, "Constructing stable grasps," Int. J. Robot. Res., vol. 8, no. 1 , pp. 26-37, 1989.

[38] J. Ponce, "On planning immobilizing fixtures for 3d polyhedral parts," in Proc. IEEE Int. Conf. Robotics Automat., 1996, pp. 509-514.

[39] P. C. Pong, R. R. Barton, and P. H. Cohen, "Optimum fixture design," in Proc. 2nd Industrial Eng. Research Conf., Los Angeles, CA, May 1993, pp. 6-10.

[40] A. Shapiro, E. Rimon, and J. W. Burdick, "On the mechanics of natural compliance in frictional contacts and its effect on grasp stiffness and stability," in Proc. IEEE Int. Conf. Robot. Automat., 2004, pp. 1264-1269.

[41] G. S. A. Shawki and M. M. Abdel-Aal, "Effect of fixture rigidity and wear on dimensional accuracy," Int. J. Mach. Tool Design Res., vol. 5, pp. $183-202,1965$.

[42] M. Teichmann, "A grasp metric invariant under rigid motions," in Proc. Int. Conf. Robot. Automat., 1996, pp. 2143-2148.
[43] S. P. Timoshenko and J. N. Goodier, Theory of Elasticity, 3rd ed. New York: McGraw-Hill, 1969.

[44] J. C. Trappey, D. S. Su, and J. L. Hou, "Computer-aided fixture analysis using finite element analysis and mathematical optimization modeling," in Proc. ASME Int. Mechanical Eng. Congr. Expo., New York, 1995, pp. 777-787.

[45] J. C. Trinkle, "On the stability and instantaneous velocity of grasped frictionless objects," IEEE Trans. Robot. Automat., vol. 8, no. 5, pp. 560-572, Oct. 1992.

[46] S. Vavasis, Nonlinear Optimization: Complexity Issues. Oxford, U.K.: Oxford Univ. Press, 1991.

[47] M. Y. Wang, "A full-kinematic model of fixtures for precision locating applications," in Proc. IEEE/RSJ Int. Conf. Intelligent Robots Systems, 2001, pp. 1135-1140.

[48] C.-H. Xiong, Y.-F. Li, H. Ding, and Y.-L. Xiong, "On the dynamic stability of grasping," Int. J. Robot. Res., vol. 18, no. 9, pp. 951-958, Sep. 1999.

[49] J. J. Xu, G. F. Liu, and Z. X. Li, "On quality functions for grasp synthesis and fixture planning," in Proc. IEEE Int. Conf. Robotics Automation, 2004, pp. 333-338.

[50] N. Xydas and I. Kao, "Modeling of contact mechanics and friction-limitsurfaces for soft fingers in robotics, with experimental results," Int. J. Robot. Res., vol. 18, no. 8, pp. 941-950, Sep. 1999.

Qiao Lin received the Ph.D. degree in mechanical engineering from the California Institute of Technology (Caltech), Pasadena, in 1998.

His doctoral research involved automated planning of robotic manipulation, with an emphasis on kinematics and mechanics of compliant fixtures. He conducted postdoctoral research in microelectromechanical systems (MEMS) with the Caltech Micromachining Laboratory from 1998 to 2000. He was an Assistant Professor with the Department of Mechanical Engineering, Carnegie Mellon University, Pittsburgh, PA from 2001 to 2004. Currently, he is an Assistant Professor with the Department of Mechanical Engineering, Columbia University, New York. His research interests are in MEMS, including micro/nanofluidic, thermal, and robotic devices for biomedical applications.

Joel W. Burdick received the B.S. degree in mechanical engineering and chemistry from Duke University, Chapel Hill, NC, and the M.S. and Ph.D. degrees in mechanical engineering from Stanford University, Stanford, CA.

Currently, he is a Professor of Mechanical Engineering and Bioengineering, the Department of Mechanical Engineering, California Institute of Technology, Pasadena, where he has been since 1988. His research interests include robotic locomotion, sensor-based robot motion planning, multifingered robotic grasping, neural prosthetics, and applied nonlinear control theory.

Dr. Burdick received the National Science Foundation Presidential Young Investigator Award (NSF PYI) from 1991 to 1995, the Office of Naval Research Young Investigator Award (ONR YI) from 1991 to 1994, and the Feynman Fellowship from 1991 to 1994.

Elon Rimon (M'96) received the B.Sc. degree in computer engineering from The Technion, Israel Institute of Technology, Haifa, Israel, in 1985, and the $\mathrm{Ph} . \mathrm{D}$. degree in electrical engineering from Yale University, New Haven, CT, in 1990.

He has been with the Department of Mechanical Engineering, Technion, since 1994, where he is currently an Associate Professor. His research interests include computational geometry algorithms in robotics, sensor-based motion planning, mobile robot problems, grasping, fixturing, and robotic locomotion. 